\title{
Respiratory mechanics models in the frame of non-local fractional operators
}

\author{
BAHAR ACAY ${ }^{a}, *(1)$, MUSTAFA INC ${ }^{a}$ (i) \\ a Department of Mathematics, Science Faculty, Firat University, 23119 Elazig, Turkey
}

- Received: 12.02.2021 • Accepted: 20.02.2021 • Published Online: 06.03.2021

\begin{abstract}
This study is intended to provide an exhaustive comparison of various non-local fractional operators when analyzing the mechanics of breathing performed by a ventilator. We present the solutions of the fractional respiratory mechanics model mainly for allowing the health care team to monitor patients' conditions in detail. The aim is to introduce the underlying model flexibly by making use of the advantages of the non-integer order operators with one, two, and three parameters. On the other hand, since we concern the usage of different types of fractional operators, most often having virtue in the application, it can be clearly observed the similarities and differences between these operators. Moreover, we observe the mobility of the solutions curves of the above-stated fractional model for different values of the parameters $\alpha, \rho$, and $\gamma$ with the help of many graphs.
\end{abstract}

Keywords: Fractional operators, Caputo derivative, Clinical medicine, Artificial respiration, Ventilator. 2010 MSC: 34A08, 26A33, 93A30.

\section{Introduction}

Fractional analysis, which has a wide range of applications in science and engineering, is an important mathematical analysis branch in which derivatives and integrals are extended to non-integer orders. The concepts of fractional derivative and fractional integral were studied in more detail in the 18th and 19th centuries, and the "paradoxes" described by Leibniz were solved by later scientists, but this does not mean that the fractional calculus is completely free from open problems. Many results in the literature reveal the advantages of the non-local fractional derivatives and integrals allowing us to observe real-world problems associated with the memory effect. The field of fractional calculus, which has been studied for centuries, has allowed many different definitions of fractional derivatives and integrals to emerge, and one of the most important definitions is the Riemann-Liouville (RL) definition. This definition for arbitrary derivative

\footnotetext{
*Corresponding author: bbahar503@gmail.com
}

(C) 2020 SABA. All Rights Reserved. 
and integral of any function emerged in the late 19th century with a complex analysis approach. Various fractional operator definitions in the literature are special cases of this general definition of RL. This crucial definition is obtained by generalizing the Cauchy integral formula under favour of the iterated derivatives of a complex analytical function, and there are several non-local fractional derivative definitions obtained from iterating some local derivatives in literature. Therefore, one of the most common and understandable ways of defining fractional calculus is to analyze the definition of RL, however, it is still not the only way to define fractional calculus. Due to the power function in the RL integral and derivative, they can be used to describe processes with the power function, but there are many different types of behavior that occur in nature and cannot be defined by simple power functions. The purpose of all other arbitrary-order operators that arise is to observe these different behaviors in nature through different types of fractional integrals and their corresponding derivatives. This clearly shows that; Fractional analysis contains various definitions instead of a specific definition in classical analysis. The fractional RL integral and derivative in [1] are defined as follows, respectively

$$
\begin{gathered}
\mathrm{RL} \mathbb{I}^{\alpha} \varphi(t)=\frac{1}{\Gamma(\alpha)} \int_{a}^{t} \frac{\varphi(\xi)}{(t-\xi)^{1-\xi}} d \xi, \quad \operatorname{Re}(\alpha)>0, \\
R L \mathbb{D}^{\alpha} \varphi(t)=\frac{d^{n}}{d t^{n}} R L \mathbb{I}^{n-\alpha} \varphi(t), \quad n=\lfloor\operatorname{Re}(\alpha)\rfloor+1, \quad \operatorname{Re}(\alpha) \geqslant 0 .
\end{gathered}
$$

In recent years, real and complex order derivatives have become a research topic that has developed not only in mathematics but also in other important areas of science such as physics, biology and engineering. Many possible definitions for fractional differentiation and integration have been proposed by making use of the RL formula and generalizations and also replacing the power function with other singular or nonsingular kernels [2]. It is possible to consider all modifications as a special case of a specific model of fractional calculus. One of the most important examples of these modifications is the Caputo fractional derivative which is more efficient than RL operator for the initial value problems. Moreover, some authors have introduced the non-local proportional operators including two parameters in [3]. This definition obtained by the iteration of the local proportional derivative based on control theory as seen in [5]. More general version of the non-local proportional operator was carried out later in [4]. Also, it can be said that the non-local proportional operator is a special case of the Prabhakar fractional operator containing generalized Mittag-Leffler function presented in 1971 [6, 7]. In [4], some authors introduced a new perspective and more general approach for proportional derivative and integral as follows:

$$
\begin{gathered}
\mathrm{PC} \mathbb{D}^{\alpha} \varphi(t)=\frac{1}{\Gamma(1-\alpha)} \int_{0}^{\mathrm{t}} \frac{\kappa_{1}(\alpha, \xi) \varphi(\xi)+\kappa_{0}(\alpha, \xi) \varphi^{\prime}(\mathrm{t})}{(\mathrm{t}-\xi)^{\alpha}} \mathrm{d} \xi, \\
\mathrm{CPC} \mathbb{D}^{\alpha} \varphi(\mathrm{t})=\frac{1}{\Gamma(1-\alpha)} \int_{0}^{\mathrm{t}} \frac{\kappa_{1}(\alpha) \varphi(\xi)+\kappa_{0}(\alpha) \varphi^{\prime}(\mathrm{t})}{(\mathrm{t}-\xi)^{\alpha}} \mathrm{d} \xi,
\end{gathered}
$$


which allows to see a combination of RL integral and Caputo derivative. Also, corresponding fractional integrals are

$$
\begin{aligned}
& \mathrm{PC}^{\alpha} \mathbb{I}^{\alpha} \varphi(t)=\int_{0}^{t} \exp \left[-\int_{u}^{t} \frac{\kappa_{1}(\alpha, \xi)}{\kappa_{0}(\alpha, \xi)} d \xi\right] \frac{R \mathrm{~L} \mathbb{D}_{\mathfrak{u}}^{1-\alpha} \varphi(u)}{\kappa_{0}(\alpha, \xi)} \mathrm{du}, \\
& \operatorname{CPC}^{\alpha} \varphi(t)=\int_{0}^{t} \exp \left[-\int_{\mathfrak{u}}^{t} \frac{\kappa_{1}(\alpha)}{\kappa_{0}(\alpha)}(t-u)\right] \frac{R_{L} \mathbb{D}_{u}^{1-\alpha} \varphi(u)}{\kappa_{0}(\alpha)} d u .
\end{aligned}
$$

One of the most important open problems in the fractional analysis is whether it is possible to create a definition that covers all the fractional operators in the literature. To make generalizations is a key point of mathematics and also many researchers studying the real-world problems in applied sciences wish to have a specific fractional operator as in the traditional analysis. Although there are many generalized fractional derivatives in the literature, there is no definition covering all fractional derivatives yet. In line with this desire, many researchers have made generalizations as an alternative to the existing fractional derivatives. In [8], authors introduced a general version of some fractional operators like RL, Caputo, Hadamard by making use of a local derivative including Mittag-Leffler function and so they get a general fractional operator with three parameters. Another generalized derivative with two parameters presented in [9]. On the other hand, the general framework of fractional operators given in [2] involves such fractional operators as RL, Prabhakar, generalized proportional, and some fractional models containing non-singular kernel [10]. In addition, authors in [11] furnished the classification of fractional calculus to comprehend the similarities or differences between the diverse fractional derivatives existing and to better understand what a fractional derivative means.

There are many different perspectives and approaches in the investigation of fractional analysis. Diverse systems and behaviors in real-world problems are not yet understood using non-integer order operators. Therefore, defining fractional derivatives without considering the applications or applying difficult conditions for all potential fractional operators will still not lead to significant progress in understanding the hidden advantages and applications of fractional analysis. On the other hand, there are various applications in the literature that have been handled with a wide variety of fractional derivatives and have better results than the classical counterparts. For example, the authors in [12] investigated the model of blood ethanol concentration model with singular and non-singular kernels by employing the real data to see the effectiveness of the fractional operators clearly. Also, some diseases and the effect of deforestation on wildlife species models analyzed in detail through non-integer order operators in $[13,14]$. Besides, since past fluctuations in the economy directly affect today's economy, fractional operators can be very effective in studying economic models owing to having the memory-effect. Hence, the authors in $[15,16]$ handled some economics models by different types of arbitrary order derivatives to observe the behavior of such models deeply. Additionally, some significant fractional applications in physics or other research areas can be seen in [17, 18, 19, 20, 21, 22, 23, 24, 25, 26].

Inspired by the above-mentioned studies, we have examined the model of respiratory mechanics in clinical medicine with various non-local singular fractional derivatives. 
The assisted ventilation is supportive care carried out with the help of the ventilator which is a crucial medical machine for the treatment of a patient. This important machine is employed in case of serious illnesses when patients lose their breathing power. The ventilator is connected to the patient with a tube in the trachea through the nose or mouth and blows air and necessary oxygen into the lung of the patient. The ventilator provides substantially more oxygen than other medical instruments like masks. Also, patients are monitored by a ventilator in order to control the respiratory rate, blood pressure, heart rate, and oxygen saturation. Thanks to the model we deal with in this study, the change in lung volume during mechanical breathing is observed by means of various non-local fractional operators with singular kernels. As can be clearly seen, the model studied is of great importance in daily life, and therefore it is thought to be useful to examine this model through different types of fractional operators such as Caputo derivative, generalized derivative, proportional derivative, $M$-derivative, and the constant proportional Caputo derivative. We focus on the fractional derivatives with a singular kernel to solve the aforementioned model and display the solution curves on the graphs without restriction with the given initial conditions. To achieve this objective, the Caputo derivative, which is very useful for initial value problems, is preferred. It is well known that other fractional derivatives with singular kernel we utilize to solve the respiratory mechanics model in the current study are modified general versions of the Caputo derivative and show very similar characteristics to each other. Hence, the similarities and differences of these fractional operators present in the literature are clearly observed through the solutions obtained with the help of Laplace transform, which is a very efficient method for constant-coefficient linear equations. Although the main derivative intended for the fractional respiratory mechanics model is the Caputo derivative, we have obtained different fractional solutions with one, two, and three parameters using some other fractional derivatives in the literature. On the other hand, we also get series solutions of the proposed model via constant proportional Caputo derivative.

We organize the current study as follows. We present the basics of fractional calculus to utilize for the main results of the paper in Section 2. Then, the description of the respiratory mechanics model and some necessary conditions are given in Section 3. Additionally, the arbitrary order solutions of the underlying model are performed by using Laplace transform (LT) in terms of Caputo fractional derivative, the generalized fractional derivative which was introduced by Katugampola, proportional derivative, non-local $M$-derivative and constant proportional Caputo derivative in this section. Finally, some essential discussions and concluding remarks are exhibited by reinforcing with graphs for different values of $\alpha, \gamma$, and $\rho$.

\section{The basics of fractional calculus}

In the current section, some crucial concepts of fractional calculus are presented for utilizing in the main results of this study.

Definition 2.1. [1] Caputo fractional derivative is defined by

$$
{ }_{C} \mathbb{D}^{\alpha} \omega(t)=\frac{1}{\Gamma(n-\alpha)} \int_{a}^{t} \frac{\omega^{(n)}(\xi)}{(t-\xi)^{1+\alpha-n}} d \xi
$$


where $\alpha \in \mathbb{C}, \operatorname{Re}(\alpha) \geqslant 0$ and $n=\lfloor\operatorname{Re}(\alpha)\rfloor+1$.

Definition 2.2. [9] The generalized fractional integral and derivative in the sense of Caputo are given respectively by

$$
\begin{array}{r}
\mathbb{I}^{\alpha, \rho} \omega(t)=\frac{1}{\Gamma(\alpha) \rho^{\alpha-1}} \int_{a}^{t} \frac{\omega(\xi)}{\left(t^{\rho}-\xi^{\rho}\right)^{1-\alpha}} \xi^{\rho-1} d \xi, \\
\mathbb{D}^{\alpha, \rho} \omega(t)=\frac{1}{\Gamma(n-\alpha) \rho^{n-\alpha-1}} \int_{a}^{t} \frac{\xi^{\rho-1}}{\left(t^{\rho}-\xi^{\rho}\right)^{1+\alpha-n}} \omega^{(n)}(\xi) d \xi,
\end{array}
$$

where $\alpha \in \mathbb{C}$ and $n=\lfloor\operatorname{Re}(\alpha)\rfloor+1$.

Definition 2.3. [3] Fractional proportional integral and derivative are defined as follows, respectively

$$
\begin{aligned}
{ }_{\mathrm{P}} \mathbb{I}^{\alpha, \rho} & \omega(t)=\frac{1}{\Gamma(\alpha) \rho^{\alpha}} \int_{a}^{t} \exp \left(\frac{\rho-1}{\rho}(t-\xi)\right)(t-\xi)^{\alpha-1} \omega(\xi) d \xi, \\
\mathrm{P}^{\alpha, \rho} \omega(t) & =D^{n, \rho} \mathbb{I}^{n-\alpha, \rho} \omega(\xi) \\
& =\frac{D^{n, \rho}}{\Gamma(n-\alpha) \rho^{n-\alpha}} \int_{a}^{t} \exp \left(\frac{\rho-1}{\rho}(t-\xi)\right)(t-\xi)^{n-\alpha-1} \omega(\xi) d \xi
\end{aligned}
$$

where $\rho \in(0,1], \alpha \in \mathbb{C}, n=\lfloor\operatorname{Re}(\alpha)\rfloor+1$ and $\mathbf{D}^{\rho}$ is the local proportional derivative given in [5].

Definition 2.4. [8] The non-local fractional M-integral and derivative are defined by

$$
\begin{aligned}
{ }_{M} \mathbb{I}^{\alpha, \rho, \gamma} \omega(t)= & \frac{\Gamma(\gamma+1)^{\alpha}}{\Gamma(\alpha) \rho^{\alpha-1}} \int_{a}^{t}\left((t-a)^{\rho}-(\xi-a)^{\rho}\right)^{\alpha-1} \omega(\xi)(\xi-a)^{\rho-1} d \xi, \\
M \mathbb{D}^{\alpha, \rho, \gamma} \omega(t) & =M \mathbb{I}^{n-\alpha, \rho, \gamma} D^{n, \rho, \gamma} \omega(t) \\
& =\frac{\Gamma(\gamma+1)^{n-\alpha}}{\Gamma(n-\alpha) \rho^{n-\alpha-1}} \int_{a}^{t}\left((t-a)^{\rho}-(\xi-a)^{\rho}\right)^{n-\alpha-1} \\
& \times D^{n, \rho, \gamma} \omega(\xi)(\xi-a)^{\rho-1} d \xi,
\end{aligned}
$$

where $\alpha \in \mathbb{C}, \gamma>0$ and $n=\lfloor\operatorname{Re}(\alpha)\rfloor+1$.

Definition 2.5. [27] The LT of the Caputo fractional derivative is defined as below

$$
\mathcal{L}_{\mathrm{a}}\left\{\mathrm{C} \mathbb{D}^{\alpha} \omega(\mathrm{t})\right\}=\mathrm{s}^{\alpha} \mathcal{W}(\mathrm{s})-\sum_{\mathrm{k}=0}^{\mathrm{n}-1} \mathrm{~s}^{\alpha-k-1} \boldsymbol{w}^{(\mathrm{k})}(\mathrm{a}),
$$

where $\mathcal{W}(s)=\mathcal{L}_{a}\{\mathfrak{w}(\mathrm{t})\}$. 
Definition 2.6. [28] Let $\omega \in A C_{\delta}^{n}[0, a], \alpha>0, s>c$ and $\delta^{k}=\left(t^{1-\rho} \frac{d}{d t}\right)^{k} \omega(t), k=$ $0,1, \ldots, n$ has exponential order $e^{\frac{\mathfrak{c} \frac{\rho}{\rho}}{\rho}}$, then the LT of generalized fractional derivative is given as follows

$$
\mathcal{L}\left\{\mathbb{D}^{\alpha, \rho} \omega(t)\right\}=s^{\alpha}\left[\mathcal{L}\{\omega(t)\}-\sum_{k=0}^{n-1} s^{-k-1}\left(\delta^{k} \omega\right)(0)\right] .
$$

Definition 2.7. [3] The LT of the non-local fractional proportional derivative is defined by

$$
\mathcal{L}_{\mathrm{a}}\left\{\mathrm{P} \mathbb{D}^{\alpha, \rho} \omega(\mathrm{t})\right\}=(\rho s+1-\rho)^{\alpha} \mathcal{W}_{\mathrm{a}}(\mathrm{s})-\rho \sum_{\mathrm{k}=0}^{\mathrm{n}-1}(\rho s+1-\rho)^{\alpha-1-k} \mathbf{D}^{\mathrm{k}, \rho} \omega(\mathrm{a}),
$$

where $\alpha \in \mathbb{C}, \operatorname{Re}(\alpha)>0, \rho \in(0,1]$ and $n=\lfloor\operatorname{Re}(\alpha)\rfloor+1$.

Definition 2.8. [8] The LT of the non-local fractional M-derivative is presented as follows

$$
\begin{aligned}
& \mathcal{L}_{\rho, \gamma}^{a}\left\{M \mathbb{D}^{n \alpha, \rho, \gamma} \omega(t)\right\}(s) \\
= & s^{\alpha} \mathcal{L}_{\rho, \gamma}^{a}\{\omega(t)\}-s^{\alpha-1} \omega(a)-s^{\alpha-2}{ }_{M} \mathbb{D}_{a}^{\rho, \gamma} \omega(a) \\
- & \ldots-s^{\alpha-n+1}{ }_{M} \mathbb{D}_{a}^{(n-2) \rho, \gamma} \omega(a)-s^{\alpha-n}{ }_{M} \mathbb{D}_{a}^{(n-1) \rho, \gamma} \omega(a),
\end{aligned}
$$

where $\omega \in \mathrm{C}_{\rho, \mathrm{a}}^{\mathrm{n}}[\mathrm{a}, \mathrm{b}], \alpha>0$ and $\gamma>0$.

Definition 2.9. [4] The LT of the constant proportional Caputo derivative is given by

$$
\mathcal{L}\left\{\mathrm{CPC} \mathbb{D}^{\alpha} \omega(\mathrm{t})\right\}=\left[\kappa_{1}(\alpha) s^{\alpha-1}+\kappa_{0}(\alpha) s^{\alpha}\right] \mathcal{L}\{\omega(t)\}-\kappa_{0}(\alpha) s^{\alpha-1} \omega(0) .
$$

\section{Description of the respiratory mechanics model and prerequisites}

Here, we intend to present the respiratory mechanics model in [29] in order to comprehend its non-integer order version. Also, we shall furnish some crucial key points of the model mentioned and the necessary assumptions for the process of mechanical breathing. This process is performed by a ventilator (medical device) and monitored by a clinician. The instantaneous volume of the lung which is modeled by a single compartment can be observed by the following differential equations with initial and end conditions:

$$
\begin{gathered}
R\left(\frac{d \mathbf{V}_{i}(t)}{d t}\right)+\left(\frac{1}{C}\right) \mathbf{V}_{i}(t)+P_{m}=P_{d}, \quad 0 \leqslant t \leqslant t_{j} \\
R\left(\frac{d \mathbf{V}_{e}(t)}{d t}\right)+\left(\frac{1}{C}\right) \mathbf{V}_{e}(t)+P_{m}=0, \quad t_{j} \leqslant t \leqslant t_{b} \\
\mathbf{V}_{\mathfrak{i}}(0)=\mathbf{V}_{e}\left(t_{b}\right)=0, \\
\mathbf{V}_{\mathfrak{i}}\left(t_{j}\right)=\mathbf{V}_{e}\left(t_{j}\right)=\mathbf{V}_{T} .
\end{gathered}
$$


The above-stated model is created by utilizing the formula of pressure balance at the airway and this pressure equation is given by

$$
\mathrm{P}_{\mathrm{l}}+\mathrm{P}_{\mathrm{k}}+\mathrm{P}_{\mathrm{m}}=\mathrm{P}_{\mathrm{a} w},
$$

where $P_{l}$ is the airway-resistance drop, that is, it is a pressure loss because of the resistance to flow into and out of the pulmonary system. Moreover, because of the volume of the lung changes during mechanical breathing, an elastic pressure occurs and this pressure is denoted by $P_{k}$. Once a breath is complete, pressure is left in the lung and it is indicated by $\mathrm{P}_{\mathrm{m}}$ called residual pressure or end-expiratory pressure. $\mathrm{P}_{\mathrm{aw}}$ stands for the pressure applied to the airway. Additionally, it should be noted that $\mathrm{P}_{\mathrm{a} w}$ is equal to the pressure $P_{d}$ during inspiration and equal to zero during expiration.

On the other hand, $t_{b}$ is the length of each breath and is determined by a clinician. Also, inspiration and expiration are two separate parts of each breath. It is presumed that inspiration occurs on $\left[0, t_{j}\right]$ and expiration occurs on $\left[t_{j}, t_{b}\right]$. Here, $t_{j}$ denotes the inspiratory time. In addition to this, $\mathrm{P}_{\mathrm{d}}$ is a pressure applied by the ventilator to the airway during inspiration and naturally $\mathrm{P}_{\mathrm{d}}=0$ during expiration. Furthermore, $\mathbf{V}_{\mathfrak{i}}(\mathrm{t})$ is the volume of the lung during inspiration on $0 \leqslant t \leqslant t_{j}, V_{e}(t)$ denotes the volume of the lung during expiration and $\mathbf{V}_{\mathrm{T}}$ stands for the tidal volume of the breath.

Due to the fact that the pressure $P_{l}$ is proportional to the flows into and out of the pulmonary system, it can be expressed that $P_{l}$ equals to $R\left(\frac{d \mathbf{V}(t)}{d t}\right)$ where $R$ is the proportionality constant. Similarly, because $P_{k}$ is proportional to the volume of lung, the elastic pressure $P_{k}$ equals $\frac{1}{C} \mathbf{V}(t)$ where $C$ is a constant called compliance of the lung. It is assumed that constants $\mathrm{R}$ and $\mathrm{C}$ are same for both inspiration and expiration.

The residual pressure $P_{m}$ can be calculated under the end condition $\mathbf{V}_{e}\left(t_{b}\right)=0$ through the following formula:

$$
P_{m}=\frac{\left(e^{t_{j} / R C}-1\right) P_{d}}{e^{t_{b} / R C}-1} .
$$

Besides, the mean alveolar pressure $\mathrm{P}_{\mathrm{ma}}$, the average pressure in the lung during inspiration, can be computed by

$$
P_{m a}=\frac{1}{C t_{j}} \int_{0}^{t_{j}} V_{i}(t) d t+P_{m}
$$

under the initial condition $\mathbf{V}_{\mathfrak{i}}(0)=0$.

Now, we furnish the fractional respiratory mechanics models by benefiting from the above pieces of information:

\subsection{Fractional respiratory mechanics model through Caputo derivative}

This portion is dedicated to interpret and describe the breathing mechanics in a mechanically ventilated patient by means of Caputo fractional derivative. Monitorization of a patient on a ventilator is crucially significant to reveal the lung function for treatment or therapy. Therefore, to utilize the non-integer order derivatives for the respiratory mechanics model can be efficient for monitoring the patient much more detailed. To that 
end, we solve this substantial model by employing the LT of Caputo fractional derivative. Let us present the fractional respiratory model via Caputo derivative as follows:

$$
\begin{gathered}
R\left[C \mathbb{D}^{\alpha} \mathbf{V}_{\mathfrak{i}}(t)\right]+\left(\frac{1}{C}\right) \mathbf{V}_{i}(t)+P_{m}=P_{d}, \quad 0 \leqslant t \leqslant t_{j}, \\
R\left[C \mathbb{D}^{\alpha} \mathbf{V}_{e}(t)\right]+\left(\frac{1}{C}\right) \mathbf{V}_{e}(t)+P_{m}=0, \quad t_{j} \leqslant t \leqslant t_{b}, \\
\mathbf{V}_{\mathfrak{i}}(0)=\mathbf{V}_{e}\left(t_{b}\right)=0, \\
\mathbf{V}_{\mathfrak{i}}\left(t_{j}\right)=\mathbf{V}_{e}\left(t_{j}\right)=\mathbf{V}_{T} .
\end{gathered}
$$

First, if we apply the LT to both side of the equation (3.8) under the condition $\mathbf{V}_{\mathfrak{i}}(0)=0$, then we have

$$
\begin{gathered}
\mathcal{L}\left\{\mathrm{R}\left[\mathrm{CD}^{\alpha} \mathbf{V}_{\mathfrak{i}}(\mathrm{t})\right]\right\}+\mathcal{L}\left\{\left(\frac{1}{\mathrm{C}}\right) \mathbf{V}_{\mathfrak{i}}(\mathrm{t})\right\}=\mathcal{L}\left\{\mathrm{P}_{\mathrm{d}}-\mathrm{P}_{\mathrm{m}}\right\}, \\
\mathrm{s}^{\alpha} \mathcal{L}\left\{\mathbf{V}_{\mathfrak{i}}(\mathrm{t})\right\}-\mathrm{s}^{\alpha-1} \mathbf{V}_{\mathfrak{i}}(0)+\left(\frac{1}{\mathrm{CR}}\right) \mathcal{L}\left\{\mathbf{V}_{\mathfrak{i}}(\mathrm{t})\right\}=\frac{\mathrm{P}_{\mathrm{d}}-\mathrm{P}_{\mathrm{m}}}{\mathrm{Rs}}, \\
\mathcal{L}\left\{\mathbf{V}_{\mathfrak{i}}(\mathrm{t})\right\}=\frac{\mathrm{P}_{\mathrm{d}}-\mathrm{P}_{\mathrm{m}}}{\mathrm{R}} \frac{1}{\mathrm{~s}\left(\mathrm{~s}^{\alpha}+\frac{1}{\mathrm{CR}}\right)},
\end{gathered}
$$

and by applying inverse LT, we can reach the following solution

$$
\mathbf{V}_{\mathfrak{i}}(\mathrm{t})=\mathrm{C}\left(\mathrm{P}_{\mathrm{d}}-\mathrm{P}_{\mathrm{m}}\right)\left[1-\mathbf{E}_{\alpha}\left(-\frac{1}{\mathrm{CR}} \mathrm{t}^{\alpha}\right)\right],
$$

where $\mathbf{E}_{\alpha}($.$) is the Mittag-Leffler function. Additionally, solving the same equation with$ the condition $\mathbf{V}_{\mathfrak{i}}\left(t_{\mathfrak{j}}\right)=\mathbf{V}_{\mathrm{T}}$, one can get the solution below

$$
\mathbf{V}_{i}(t)=C\left(P_{d}-P_{m}\right)\left[1-E_{\alpha}\left(-\frac{1}{C R}\left(t-t_{j}\right)^{\alpha}\right)\right]+\mathbf{V}_{T}\left[E_{\alpha}\left(-\frac{1}{C R}\left(t-t_{j}\right)^{\alpha}\right)\right] .
$$

Now, we solve the equation (3.9) under the condition $\mathbf{V}_{\boldsymbol{e}}\left(\mathbf{t}_{\mathbf{b}}\right)=0$. Taking the LT of (3.9), we obtain

$$
\begin{gathered}
\mathcal{L}\left\{R\left[C \mathbb{D}^{\alpha} \mathbf{V}_{e}(t)\right]\right\}+\mathcal{L}\left\{\left(\frac{1}{C}\right) \mathbf{V}_{e}(t)\right\}=\mathcal{L}\left\{-P_{m}\right\} \\
s^{\alpha} \mathcal{L}\left\{\mathbf{V}_{e}(t)\right\}-s^{\alpha-1} \mathbf{V}_{e}\left(t_{b}\right)+\left(\frac{1}{C R}\right) \mathcal{L}\left\{\mathbf{V}_{e}(t)\right\}=\frac{-P_{m}}{R s} \\
\mathcal{L}\left\{\mathbf{V}_{e}(t)\right\}=\frac{-P_{m}}{R} \frac{1}{s\left(s^{\alpha}+\frac{1}{C R}\right)},
\end{gathered}
$$


applying inverse LT, we can readily attain the following solution

$$
\mathbf{V}_{e}(t)=-C P_{m}\left[1-E_{\alpha}\left(-\frac{1}{C R}\left(t-t_{b}\right)^{\alpha}\right)\right] .
$$

In a similar manner, if we solve the equation (3.9) with the end condition $\mathbf{V}_{e}\left(t_{j}\right)=\mathbf{V}_{T}$, we have

$$
\mathbf{V}_{e}(t)=-C P_{m}\left[1-E_{\alpha}\left(-\frac{1}{C R}\left(t-t_{j}\right)^{\alpha}\right)\right]+\mathbf{V}_{T}\left[E_{\alpha}\left(-\frac{1}{C R}\left(t-t_{j}\right)^{\alpha}\right)\right] .
$$

\subsection{Fractional respiratory mechanics model through constant proportional Caputo derivative}

Here, we consider and analyze the respiratory mechanics model in the frame of the constant proportional Caputo (CPC) derivative to identify the instantaneous volume of the lung. Fractional version of the model (3.8)-(3.9) can be presented by CPC derivative as follows

$$
\begin{gathered}
R\left[\mathrm{CPC}^{\alpha} \mathbf{V}_{\mathfrak{i}}(t)\right]+\left(\frac{1}{\mathrm{C}}\right) \mathbf{V}_{\mathfrak{i}}(t)+P_{m}=P_{d}, \quad 0 \leqslant t \leqslant t_{j}, \\
R\left[\mathrm{CPC}^{\alpha} \mathbf{V}_{e}(t)\right]+\left(\frac{1}{C}\right) \mathbf{V}_{e}(t)+P_{m}=0, \quad t_{j} \leqslant t \leqslant t_{b}, \\
\mathbf{V}_{i}(0)=\mathbf{V}_{e}\left(t_{b}\right)=0, \\
\mathbf{V}_{i}\left(t_{j}\right)=\mathbf{V}_{e}\left(t_{j}\right)=\mathbf{V}_{T} .
\end{gathered}
$$

Begin with solving the equation (3.22) under the initial condition $\mathbf{V}_{\mathfrak{i}}(0)=0$ by virtue of the LT, we get

$$
\begin{aligned}
& \mathcal{L}\left\{\mathrm{R}\left[\mathrm{CPC} \mathbb{D}^{\alpha} \mathbf{V}_{\mathrm{i}}(\mathrm{t})\right]\right\}+\mathcal{L}\left\{\left(\frac{1}{\mathrm{C}}\right) \mathbf{V}_{\mathrm{i}}(\mathrm{t})\right\}=\mathcal{L}\left\{\mathrm{P}_{\mathrm{d}}-\mathrm{P}_{\mathrm{m}}\right\} \\
& {\left[\kappa_{1}(\alpha) s^{\alpha-1}+\kappa_{0}(\alpha) s^{\alpha}\right] \mathcal{L}\left\{\mathbf{V}_{\mathfrak{i}}(t)\right\}-\kappa_{0}(\alpha) s^{\alpha-1} \mathbf{V}_{\mathfrak{i}}(0)+\frac{1}{R C} \mathcal{L}\left\{\mathbf{V}_{\mathfrak{i}}(t)\right\}=\frac{P_{d}-P_{m}}{R s}} \\
& \mathcal{L}\left\{\mathbf{V}_{\mathfrak{i}}(t)\right\}=\frac{\mathrm{P}_{\mathrm{d}}-\mathrm{P}_{\mathrm{m}}}{\mathrm{R}\left[\mathrm{K}_{1}(\alpha) \mathrm{s}^{\alpha}+\mathrm{K}_{0}(\alpha) \mathrm{s}^{\alpha+1}+\frac{\mathrm{s}}{\mathrm{RC}}\right]}, \\
& \mathcal{L}\left\{\mathbf{V}_{\mathfrak{i}}(\mathrm{t})\right\}=\mathrm{C}\left(\mathrm{P}_{\mathrm{d}}-\mathrm{P}_{\mathrm{m}}\right) \mathrm{s}^{-1} \frac{1}{1-\left[-\mathrm{RC}_{\kappa_{1}}(\alpha) \mathrm{s}^{\alpha-1}-\mathrm{RC} \kappa_{0}(\alpha) \mathrm{s}^{\alpha}\right]},
\end{aligned}
$$

and if we write in serial form, we have

$$
\mathcal{L}\left\{\mathbf{V}_{\mathfrak{i}}(\mathrm{t})\right\}=\mathrm{C}\left(\mathrm{P}_{\mathrm{d}}-\mathrm{P}_{\mathrm{m}}\right) \mathrm{s}^{-1} \sum_{\mathrm{k}=0}^{\infty}\left[-\mathrm{RC} \mathrm{k}_{1}(\alpha) \mathrm{s}^{\alpha-1}-\mathrm{RC} \mathrm{k}_{0}(\alpha) \mathrm{s}^{\alpha}\right]^{\mathrm{k}},
$$




$$
\begin{aligned}
& \mathcal{L}\left\{\mathbf{V}_{i}(t)\right\}=C\left(P_{d}-P_{m}\right) s^{-1} \sum_{k=0}^{\infty} \frac{1}{R^{k} C^{k}} \sum_{n=0}^{k}\left(\begin{array}{l}
k \\
n
\end{array}\right)\left[-\kappa_{1}(\alpha) s^{\alpha-1}\right]^{k-n}\left[-\kappa_{0}(\alpha) s^{\alpha}\right]^{n}, \\
& \mathcal{L}\left\{\mathbf{V}_{\mathrm{i}}(\mathrm{t})\right\}=\mathrm{C}\left(\mathrm{P}_{\mathrm{d}}-\mathrm{P}_{\mathrm{m}}\right) \sum_{\mathrm{k}=0}^{\infty} \sum_{n=0}^{\mathrm{k}} \frac{(-1)^{\mathrm{k}}\left(\mathrm{k}_{1}(\alpha)\right)^{\mathrm{k}-\mathrm{n}}\left(\mathrm{k}_{0}(\alpha)\right)^{\mathrm{n}}}{\mathrm{R}^{\mathrm{k}} \mathrm{C}^{\mathrm{k}}}\left(\begin{array}{l}
\mathrm{k} \\
\mathrm{n}
\end{array}\right) \mathrm{s}^{(\alpha-1)(\mathrm{k}-\mathrm{n})+\alpha n-1},
\end{aligned}
$$

applying inverse Laplace transform to the equation (3.32), one can obtain

$$
\mathbf{V}_{i}(t)=C\left(P_{d}-P_{m}\right) \sum_{k=0}^{\infty} \sum_{n=0}^{k}(-1)^{k} \frac{\left(k_{1}(\alpha)\right)^{k-n}\left(k_{0}(\alpha)\right)^{n}}{R^{k} C^{k}}\left(\begin{array}{l}
k \\
n
\end{array}\right) \frac{t^{(1-\alpha) k-n}}{\Gamma((1-\alpha) k-n+1)}
$$

For $\lambda=k-n$, we reach

$$
\begin{gathered}
\mathbf{V}_{\mathfrak{i}}(t)=C\left(P_{d}-P_{m}\right) \sum_{k=0}^{\infty} \sum_{n=0}^{k} \frac{(n+\lambda) !}{n ! \lambda !} \frac{\left(-\kappa_{1}(\alpha)\right)^{\lambda}\left(-k_{0}(\alpha)\right)^{n}}{R^{\lambda+n} C^{\lambda+n}} \frac{t^{(1-\alpha) \lambda-\alpha n}}{\Gamma((1-\alpha) \lambda-\alpha n+1)}, \\
\mathbf{V}_{i}(t)=C\left(P_{d}-P_{m}\right) \sum_{k=0}^{\infty} \sum_{n=0}^{\infty} \frac{(n+\lambda) !}{n ! \lambda !}\left[\frac{-k_{0}(\alpha)}{R C} t^{-\alpha}\right]^{n}\left[\frac{-\kappa_{1}(\alpha)}{R C} t^{1-\alpha}\right]^{\lambda} \frac{1}{\Gamma((1-\alpha) \lambda-\alpha n+1)},
\end{gathered}
$$

By rewriting the equation (3.35) with the help of Mittag-Leffler function as seen in [30], we get the following solution

$$
\mathbf{V}_{\mathfrak{i}}(\mathrm{t})=\mathrm{C}\left(\mathrm{P}_{\mathrm{d}}-\mathrm{P}_{\mathrm{m}}\right) \mathbf{E}_{1-\alpha,-\alpha, 1}^{1}\left(\frac{-\mathrm{K}_{1}(\alpha)}{\mathrm{RC}} \mathrm{t}^{1-\alpha}, \frac{-\mathrm{K}_{0}(\alpha)}{\mathrm{RC}} \mathrm{t}^{-\alpha}\right) .
$$

If we solve the equation (3.22) under the condition $\mathbf{V}_{\mathfrak{i}}\left(t_{j}\right)=\mathbf{V}_{\mathrm{T}}$, we attain as below

$$
\begin{aligned}
& \mathcal{L}\left\{R\left[C P C \mathbb{D}^{\alpha} \mathbf{V}_{\mathfrak{i}}(\mathrm{t})\right]\right\}+\mathcal{L}\left\{\left(\frac{1}{\mathrm{C}}\right) \mathbf{V}_{\mathfrak{i}}(\mathrm{t})\right\}=\mathcal{L}\left\{\mathrm{P}_{\mathrm{d}}-\mathrm{P}_{\mathrm{m}}\right\}, \\
& {\left[\kappa_{1}(\alpha) s^{\alpha-1}+\kappa_{0}(\alpha) s^{\alpha}\right] \mathcal{L}\left\{\mathbf{V}_{\mathfrak{i}}(t)\right\}-\kappa_{0}(\alpha) s^{\alpha-1} \mathbf{V}_{\mathrm{T}}+\frac{1}{C R} \mathcal{L}\left\{\mathbf{V}_{\mathfrak{i}}(\mathrm{t})\right\}=\frac{\mathrm{P}_{\mathrm{d}}-\mathrm{P}_{\mathrm{m}}}{\mathrm{Rs}},} \\
& \mathcal{L}\left\{\mathbf{V}_{\mathfrak{i}}(t)\right\}=\frac{\mathrm{P}_{\mathrm{d}}-\mathrm{P}_{\mathrm{m}}}{\mathrm{R}\left[\mathrm{K}_{1}(\alpha) \mathrm{s}^{\alpha}+\mathrm{K}_{0}(\alpha) \mathrm{s}^{\alpha+1}+\frac{\mathrm{s}}{\mathrm{RC}}\right]}+\frac{\mathrm{RCV}_{\mathrm{T}} \kappa_{0}(\alpha) \mathrm{s}^{\alpha-1}}{R C \kappa_{1}(\alpha) s^{\alpha-1}+R C \kappa_{0}(\alpha) s^{\alpha}+1}, \\
& \mathcal{L}\left\{\mathbf{V}_{\mathfrak{i}}(\mathrm{t})\right\}=\mathrm{C}\left(\mathrm{P}_{\mathrm{d}}-\mathrm{P}_{\mathrm{m}}\right) \mathrm{s}^{-1} \frac{1}{1-\left[-\mathrm{RC} \kappa_{1}(\alpha) \mathrm{s}^{\alpha-1}-\mathrm{RC}_{\mathrm{K}}(\alpha) \mathrm{s}^{\alpha}\right]} \\
& +\mathbf{V}_{\mathrm{T}} \mathrm{s}^{-1} \frac{1}{1-\left[\frac{-R \mathrm{~K}_{1}(\alpha) s^{-1}-\mathrm{s}^{-\alpha}}{\mathrm{RC} \kappa_{0}(\alpha)}\right]},
\end{aligned}
$$




$$
\begin{aligned}
& \mathcal{L}\left\{\mathbf{V}_{\mathfrak{i}}(t)\right\}=C\left(P_{d}-P_{m}\right) s^{-1} \sum_{k=0}^{\infty}\left[-R C \kappa_{1}(\alpha) s^{\alpha-1}-R C \kappa_{0}(\alpha) s^{\alpha}\right]^{k} \\
& +\mathbf{V}_{\mathrm{T}} \mathrm{s}^{-1} \sum_{\mathrm{k}=0}^{\infty}\left[\frac{-\mathrm{RC} \kappa_{1}(\alpha) \mathrm{s}^{-1}-\mathrm{s}^{-\alpha}}{\mathrm{RC} \mathrm{K}_{0}(\alpha)}\right]^{\mathrm{k}}, \\
& \mathcal{L}\left\{\mathbf{V}_{\mathfrak{i}}(\mathrm{t})\right\}=\mathrm{C}\left(\mathrm{P}_{\mathrm{d}}-\mathrm{P}_{\mathrm{m}}\right) \mathrm{s}^{-1} \sum_{\mathrm{k}=0}^{\infty} \frac{1}{\mathrm{R}^{\mathrm{k}} \mathrm{C}^{\mathrm{k}}} \sum_{\mathrm{n}=0}^{\mathrm{k}}\left(\begin{array}{l}
\mathrm{k} \\
\mathrm{n}
\end{array}\right)\left[-\mathrm{k}_{1}(\alpha) \mathrm{s}^{\alpha-1}\right]^{\mathrm{k}-\mathrm{n}}\left[-\mathrm{k}_{0}(\alpha) \mathrm{s}^{\alpha}\right]^{\mathrm{n}} \\
& +\mathbf{V}_{\mathrm{T}} s^{-1} \sum_{k=0}^{\infty} \frac{1}{R^{k} C^{k}\left(\kappa_{0}(\alpha)^{k}\right)} \sum_{n=0}^{k}\left(\begin{array}{l}
k \\
n
\end{array}\right)\left[-R C \kappa_{1}(\alpha) s^{-1}\right]^{k-n}\left[-s^{-\alpha}\right]^{n}, \\
& \mathcal{L}\left\{\mathbf{V}_{\mathfrak{i}}(\mathrm{t})\right\}=\mathrm{C}\left(\mathrm{P}_{\mathrm{d}}-\mathrm{P}_{\mathrm{m}}\right) \sum_{\mathrm{k}=0}^{\infty} \sum_{n=0}^{\mathrm{k}} \frac{(-1)^{\mathrm{k}}\left(\mathrm{k}_{1}(\alpha)\right)^{\mathrm{k}-\mathrm{n}}\left(\mathrm{k}_{0}(\alpha)\right)^{\mathrm{n}}}{\mathrm{R}^{\mathrm{k}} \mathrm{C}^{\mathrm{k}}}\left(\begin{array}{l}
\mathrm{k} \\
\mathrm{n}
\end{array}\right) \mathrm{s}^{(\alpha-1)(\mathrm{k}-\mathrm{n})+\alpha n-1} \\
& +\mathbf{V}_{\mathrm{T}} \sum_{k=0}^{\infty} \sum_{n=0}^{k}(-1)^{k} \frac{R^{k-n} C^{k-n}\left(\kappa_{1}(\alpha)\right)^{k-n}}{R^{k} C^{k}\left(\kappa_{0}(\alpha)\right)^{k}}\left(\begin{array}{l}
k \\
n
\end{array}\right) s^{k-n-\alpha n-1} .
\end{aligned}
$$

If we implement the inverse $\mathrm{LT}$, then we can get

$$
\begin{aligned}
\mathbf{V}_{i}(t) & =C\left(P_{d}-P_{m}\right) \sum_{k=0}^{\infty} \sum_{n=0}^{k}(-1)^{k} \frac{\left(k_{1}(\alpha)\right)^{k-n}\left(k_{0}(\alpha)\right)^{n}}{R^{k} C^{k}}\left(\begin{array}{c}
k \\
n
\end{array}\right) \frac{\left(t-t_{j}\right)^{(1-\alpha) k-n}}{\Gamma((1-\alpha) k-n+1)} \\
& +\mathbf{V}_{T} \sum_{k=0}^{\infty} \sum_{n=0}^{k}(-1)^{k} \frac{R^{k-n} C^{k-n}\left(k_{1}(\alpha)\right)^{k-n}}{R^{k} C^{k}\left(k_{0}(\alpha)\right)^{k}}\left(\begin{array}{l}
k \\
n
\end{array}\right) \frac{\left(t-t_{j}\right)^{k+(\alpha-1) n}}{\Gamma(k+(\alpha-1) n+1)},(3.44)
\end{aligned}
$$

taking $\lambda=k-n$, one can have

$$
\begin{aligned}
\mathbf{V}_{i}(t) & =C\left(P_{d}-P_{m}\right) \sum_{k=0}^{\infty} \sum_{n=0}^{k} \frac{(n+\lambda) !}{n ! \lambda !} \frac{\left(-k_{1}(\alpha)\right)^{\lambda}\left(-k_{0}(\alpha)\right)^{n}}{R^{\lambda+n} C^{\lambda+n}} \frac{\left(t-t_{j}\right)^{(1-\alpha) \lambda-\alpha n}}{\Gamma((1-\alpha) \lambda-\alpha n+1)} \\
& +\mathbf{V}_{T} \sum_{k=0}^{\infty} \sum_{n=0}^{k} \frac{(n+\lambda) !}{n ! \lambda !} \frac{R^{\lambda} C^{\lambda}\left(-k_{1}(\alpha)\right)^{\lambda}(-1)^{n}}{R^{\lambda+n} C^{\lambda+n}\left(\kappa_{0}(\alpha)\right)^{\lambda+n}} \frac{\left(t-t_{j}\right)^{\lambda+\alpha n}}{\Gamma(\lambda+\alpha n+1)}
\end{aligned}
$$




$$
\begin{aligned}
\mathbf{V}_{i}(t) & =C\left(P_{d}-P_{m}\right) \sum_{k=0}^{\infty} \sum_{n=0}^{\infty} \frac{(n+\lambda) !}{n ! \lambda !}\left[\frac{-\kappa_{0}(\alpha)}{R C}\left(t-t_{j}\right)^{-\alpha}\right]^{n} \\
& \times\left[\frac{-\kappa_{1}(\alpha)}{R C}\left(t-t_{j}\right)^{1-\alpha}\right]^{\lambda} \frac{1}{\Gamma((1-\alpha) \lambda-\alpha n+1)} \\
& +V_{T} \sum_{k=0}^{\infty} \sum_{n=0}^{\infty} \frac{(n+\lambda) !}{n ! \lambda !}\left[\frac{-1}{R C \kappa_{0}(\alpha)}\left(t-t_{j}\right)^{\alpha}\right]^{n}\left[\frac{-\kappa_{1}(\alpha)}{\kappa_{0}(\alpha)}\left(t-t_{j}\right)\right]^{\lambda} \frac{1}{\Gamma(\lambda+\alpha n+1)}
\end{aligned}
$$

and so we obtain the following solution

$$
\begin{aligned}
\mathbf{V}_{i}(t) & =C\left(P_{d}-P_{m}\right) E_{1-\alpha,-\alpha, 1}^{1}\left(\frac{-\kappa_{1}(\alpha)}{R C}\left(t-t_{j}\right)^{1-\alpha}, \frac{-k_{0}(\alpha)}{R C}\left(t-t_{j}\right)^{-\alpha}\right) \\
& +V_{T} E_{1, \alpha, 1}^{1}\left(\frac{-\kappa_{1}(\alpha)}{\kappa_{0}(\alpha)}\left(t-t_{j}\right), \frac{-1}{R C \kappa_{0}(\alpha)}\left(t-t_{j}\right)^{\alpha}\right) .
\end{aligned}
$$

Now, let us solve the equation (3.23) under the condition $\mathbf{V}_{\boldsymbol{e}}\left(\mathrm{t}_{\mathrm{b}}\right)=0$ with the help of LT as below

$$
\begin{aligned}
& \mathcal{L}\left\{R\left[C P C \mathbb{D}^{\alpha} \mathbf{V}_{e}(t)\right]\right\}+\mathcal{L}\left\{\left(\frac{1}{C}\right) \mathbf{V}_{e}(t)\right\}=\mathcal{L}\left\{-P_{m}\right\}, \\
& {\left[\kappa_{1}(\alpha) s^{\alpha-1}+\kappa_{0}(\alpha) s^{\alpha}\right] \mathcal{L}\left\{\mathbf{V}_{e}(t)\right\}-\kappa_{0}(\alpha) s^{\alpha-1} \mathbf{V}_{e}\left(t_{b}\right)+\frac{1}{R C} \mathcal{L}\left\{\mathbf{V}_{\mathfrak{i}}(t)\right\}=\frac{-P_{m}}{R s},} \\
& \mathcal{L}\left\{\mathbf{V}_{\mathrm{e}}(\mathrm{t})\right\}=\frac{-\mathrm{P}_{\mathrm{m}}}{\mathrm{R}\left[\mathrm{\kappa}_{1}(\alpha) \mathrm{s}^{\alpha}+\mathrm{\kappa}_{0}(\alpha) \mathrm{s}^{\alpha+1}+\frac{\mathrm{s}}{\mathrm{RC}}\right]}, \\
& \mathcal{L}\left\{\mathbf{V}_{\mathrm{e}}(\mathrm{t})\right\}=-\mathrm{CP}_{\mathrm{m}} \mathrm{s}^{-1} \frac{1}{1-\left[-\mathrm{RC}_{\mathrm{K}_{1}}(\alpha) \mathrm{s}^{\alpha-1}-\mathrm{RC}_{\left.\mathrm{K}_{0}(\alpha) s^{\alpha}\right]^{\prime}}\right.},
\end{aligned}
$$

and by writing in serial form, we can get

$$
\begin{gathered}
\mathcal{L}\left\{\mathbf{V}_{e}(t)\right\}=-C_{m} s^{-1} \sum_{k=0}^{\infty}\left[-R C \kappa_{1}(\alpha) s^{\alpha-1}-R C \kappa_{0}(\alpha) s^{\alpha}\right]^{k}, \\
\mathcal{L}\left\{\mathbf{V}_{e}(t)\right\}=-C P_{m} s^{-1} \sum_{k=0}^{\infty} \frac{1}{R^{k} C^{k}} \sum_{n=0}^{k}\left(\begin{array}{c}
k \\
n
\end{array}\right)\left[-\kappa_{1}(\alpha) s^{\alpha-1}\right]^{k-n}\left[-\kappa_{0}(\alpha) s^{\alpha}\right]^{n}, \\
\mathcal{L}\left\{\mathbf{V}_{e}(t)\right\}=-C P_{m} \sum_{k=0}^{\infty} \sum_{n=0}^{k} \frac{(-1)^{k}\left(\kappa_{1}(\alpha)\right)^{k-n}\left(\kappa_{0}(\alpha)\right)^{n}}{R^{k} C^{k}}\left(\begin{array}{c}
k \\
n
\end{array}\right) s^{(\alpha-1)(k-n)+\alpha n-1},
\end{gathered}
$$


if we take inverse Laplace transform of the equation (3.54), we obtain

$$
\mathbf{V}_{e}(t)=-C_{m} \sum_{k=0}^{\infty} \sum_{n=0}^{k}(-1)^{k} \frac{\left(k_{1}(\alpha)\right)^{k-n}\left(k_{0}(\alpha)\right)^{n}}{R^{k} C^{k}}\left(\begin{array}{l}
k \\
n
\end{array}\right) \frac{\left(t-t_{b}\right)^{(1-\alpha) k-n}}{\Gamma((1-\alpha) k-n+1)} .
$$

For $\lambda=k-n$, we get

$$
\begin{aligned}
\mathbf{V}_{e}(t)= & -C P_{m} \sum_{k=0}^{\infty} \sum_{n=0}^{k} \frac{(n+\lambda) !}{n ! \lambda !} \frac{\left(-\kappa_{1}(\alpha)\right)^{\lambda}\left(-k_{0}(\alpha)\right)^{n}}{R^{\lambda+n} C^{\lambda+n}} \frac{\left(t-t_{b}\right)^{(1-\alpha) \lambda-\alpha n}}{\Gamma((1-\alpha) \lambda-\alpha n+1)} \\
\mathbf{V}_{e}(t) & =-C_{m} \sum_{k=0}^{\infty} \sum_{n=0}^{\infty} \frac{(n+\lambda) !}{n ! \lambda !}\left[\frac{-\kappa_{0}(\alpha)}{R C}\left(t-t_{b}\right)^{-\alpha}\right]^{n}\left[\frac{-\kappa_{1}(\alpha)}{R C}\left(t-t_{b}\right)^{1-\alpha}\right]^{\lambda} \\
& \times \frac{1}{\Gamma((1-\alpha) \lambda-\alpha n+1)}
\end{aligned}
$$

rewriting the equation (3.57) with the help of Mittag-Leffler function as seen in [30], we have the following solution

$$
\mathbf{V}_{e}(t)=-C P_{m} E_{1-\alpha,-\alpha, 1}^{1}\left(\frac{-k_{1}(\alpha)}{R C}\left(t-t_{b}\right)^{1-\alpha}, \frac{-k_{0}(\alpha)}{R C}\left(t-t_{b}\right)^{-\alpha}\right) .
$$

Similarly, if we solve the equation (3.23) with the condition $\mathbf{V}_{\boldsymbol{e}}\left(\mathbf{t}_{\mathbf{j}}\right)=\mathbf{V}_{\mathrm{T}}$, we have

$$
\begin{gathered}
\mathcal{L}\left\{R\left[C P C \mathbb{D}^{\alpha} \mathbf{V}_{e}(t)\right]\right\}+\mathcal{L}\left\{\left(\frac{1}{C}\right) \mathbf{V}_{e}(t)\right\}=\mathcal{L}\left\{-P_{m}\right\}, \\
{\left[\kappa_{1}(\alpha) s^{\alpha-1}+\kappa_{0}(\alpha) s^{\alpha}\right] \mathcal{L}\left\{\mathbf{V}_{e}(t)\right\}-\kappa_{0}(\alpha) s^{\alpha-1} \mathbf{V}_{\mathrm{T}}+\frac{1}{C R} \mathcal{L}\left\{\mathbf{V}_{e}(t)\right\}=\frac{-P_{m}}{R s},} \\
\mathcal{L}\left\{\mathbf{V}_{e}(t)\right\}=\frac{-P_{m}}{R\left[k_{1}(\alpha) s^{\alpha}+\kappa_{0}(\alpha) s^{\alpha+1}+\frac{s}{R C}\right]}+\frac{R C V_{T} k_{0}(\alpha) s^{\alpha-1}}{R C \kappa_{1}(\alpha) s^{\alpha-1}+R C \kappa_{0}(\alpha) s^{\alpha}+1}, \\
\mathcal{L}\left\{\mathbf{V}_{e}(t)\right\}=-C P_{m} s^{-1} \frac{1}{1-\left[-R C \kappa_{1}(\alpha) s^{\alpha-1}-R C \kappa_{0}(\alpha) s^{\alpha}\right]} \\
+\mathbf{V}_{\mathrm{T}} s^{-1} \frac{1}{1-\left[\frac{-R C \kappa_{1}(\alpha) s^{-1}-s^{-\alpha}}{R C \kappa_{0}(\alpha)}\right.}, \\
\mathcal{L}\left\{\mathbf{V}_{e}(t)\right\}=-C P_{m} s^{-1} \sum_{k=0}^{\infty}\left[-R C \kappa_{1}(\alpha) s^{\alpha-1}-R C \kappa_{0}(\alpha) s^{\alpha}\right]^{k} \\
+\mathbf{V}_{\mathrm{T}} s^{-1} \sum_{k=0}^{\infty}\left[\frac{-R C \kappa_{1}(\alpha) s^{-1}-s^{-\alpha}}{R C \kappa_{0}(\alpha)}\right]^{k},
\end{gathered}
$$




$$
\begin{aligned}
& \mathcal{L}\left\{\mathbf{V}_{e}(t)\right\}=-\mathrm{CP}_{\mathrm{m}} \mathrm{s}^{-1} \sum_{\mathrm{k}=0}^{\infty} \frac{1}{\mathrm{R}^{\mathrm{k}} \mathrm{C}^{\mathrm{k}}} \sum_{\mathrm{n}=0}^{\mathrm{k}}\left(\begin{array}{l}
\mathrm{k} \\
\mathrm{n}
\end{array}\right)\left[-\mathrm{\kappa}_{1}(\alpha) \mathrm{s}^{\alpha-1}\right]^{\mathrm{k}-\mathrm{n}}\left[-\mathrm{\kappa}_{0}(\alpha) \mathrm{s}^{\alpha}\right]^{\mathrm{n}} \\
& +\quad \mathbf{V}_{\mathrm{T}} \mathrm{s}^{-1} \sum_{\mathrm{k}=0}^{\infty} \frac{1}{\mathrm{R}^{\mathrm{k}} \mathrm{C}^{\mathrm{k}}\left(\mathrm{K}_{0}(\alpha)^{\mathrm{k}}\right)} \sum_{n=0}^{k}\left(\begin{array}{l}
k \\
n
\end{array}\right)\left[-R \mathrm{C}_{1}(\alpha) \mathrm{s}^{-1}\right]^{\mathrm{k}-\mathrm{n}}\left[-\mathrm{s}^{-\alpha}\right]^{\mathrm{n}}, \\
& \mathcal{L}\left\{\mathbf{V}_{e}(t)\right\}=-C P_{m} \sum_{k=0}^{\infty} \sum_{n=0}^{k} \frac{(-1)^{k}\left(\kappa_{1}(\alpha)\right)^{k-n}\left(\kappa_{0}(\alpha)\right)^{n}}{R^{k} C^{k}}\left(\begin{array}{l}
k \\
n
\end{array}\right) s^{(\alpha-1)(k-n)+\alpha n-1} \\
& +\mathbf{V}_{\mathrm{T}} \sum_{\mathrm{k}=0}^{\infty} \sum_{n=0}^{\mathrm{k}}(-1)^{\mathrm{k}} \frac{\mathrm{R}^{\mathrm{k}-\mathrm{n}} \mathrm{C}^{\mathrm{k}-\mathrm{n}}\left(\mathrm{k}_{1}(\alpha)\right)^{\mathrm{k}-\mathrm{n}}}{\mathrm{R}^{\mathrm{k}} \mathrm{C}^{\mathrm{k}}\left(\mathrm{k}_{0}(\alpha)\right)^{\mathrm{k}}}\left(\begin{array}{l}
\mathrm{k} \\
\mathrm{n}
\end{array}\right) \mathrm{s}^{\mathrm{k}-\mathrm{n}-\alpha \mathrm{n}-1} \text {. }
\end{aligned}
$$

After applying the inverse LT, we attain

$$
\begin{aligned}
\mathbf{V}_{e}(t) & =-C P_{m} \sum_{k=0}^{\infty} \sum_{n=0}^{k}(-1)^{k} \frac{\left(k_{1}(\alpha)\right)^{k-n}\left(k_{0}(\alpha)\right)^{n}}{R^{k} C^{k}}\left(\begin{array}{l}
k \\
n
\end{array}\right) \frac{\left(t-t_{j}\right)^{(1-\alpha) k-n}}{\Gamma((1-\alpha) k-n+1)} \\
& +\mathbf{V}_{T} \sum_{k=0}^{\infty} \sum_{n=0}^{k}(-1)^{k} \frac{R^{k-n} C^{k-n}\left(k_{1}(\alpha)\right)^{k-n}}{R^{k} C^{k}\left(k_{0}(\alpha)\right)^{k}}\left(\begin{array}{l}
k \\
n
\end{array}\right) \frac{\left(t-t_{j}\right)^{k+(\alpha-1) n}}{\Gamma(k+(\alpha-1) n+1)^{\prime}}
\end{aligned}
$$

for $\lambda=k-n$,

$$
\begin{aligned}
\mathbf{V}_{e}(t) & =-C P_{m} \sum_{k=0}^{\infty} \sum_{n=0}^{k} \frac{(n+\lambda) !}{n ! \lambda !} \frac{\left(-\kappa_{1}(\alpha)\right)^{\lambda}\left(-\kappa_{0}(\alpha)\right)^{n}}{R^{\lambda+n} C^{\lambda+n}} \frac{\left(t-t_{j}\right)^{(1-\alpha) \lambda-\alpha n}}{\Gamma((1-\alpha) \lambda-\alpha n+1)}(3.67) \\
& +\mathbf{V}_{T} \sum_{k=0}^{\infty} \sum_{n=0}^{k} \frac{(n+\lambda) !}{n ! \lambda !} \frac{R^{\lambda} C^{\lambda}\left(-\kappa_{1}(\alpha)\right)^{\lambda}(-1)^{n}}{R^{\lambda+n} C^{\lambda+n}\left(\kappa_{0}(\alpha)\right)^{\lambda+n}} \frac{\left(t-t_{j}\right)^{\lambda+\alpha n}}{\Gamma(\lambda+\alpha n+1)^{\prime}}, \\
\mathbf{V}_{e}(t) & =-C P_{m} \sum_{k=0}^{\infty} \sum_{n=0}^{\infty} \frac{(n+\lambda) !}{n ! \lambda !}\left[\frac{-\kappa_{0}(\alpha)}{R C}\left(t-t_{j}\right)^{-\alpha}\right]^{n} \\
& \times\left[\frac{-\kappa_{1}(\alpha)}{R C}\left(t-t_{j}\right)^{1-\alpha}\right]^{\lambda} \frac{1}{\Gamma((1-\alpha) \lambda-\alpha n+1)} \\
& +V_{T} \sum_{k=0}^{\infty} \sum_{n=0}^{\infty} \frac{(n+\lambda) !}{n ! \lambda !}\left[\frac{-1}{R C \kappa_{0}(\alpha)}\left(t-t_{j}\right)^{\alpha}\right]^{n}\left[\frac{-\kappa_{1}(\alpha)}{k_{0}(\alpha)}\left(t-t_{j}\right)\right]^{\lambda} \frac{1}{\Gamma(\lambda+\alpha n+1)},
\end{aligned}
$$

and hence we get the desired solution as follows 


$$
\begin{aligned}
\mathbf{V}_{e}(t) & =-C P_{m} E_{1-\alpha,-\alpha, 1}^{1}\left(\frac{-\kappa_{1}(\alpha)}{R C}\left(t-t_{j}\right)^{1-\alpha}, \frac{-\kappa_{0}(\alpha)}{R C}\left(t-t_{j}\right)^{-\alpha}\right) \\
& +V_{T} E_{1, \alpha, 1}^{1}\left(\frac{-\kappa_{1}(\alpha)}{\kappa_{0}(\alpha)}\left(t-t_{j}\right), \frac{-1}{R C \kappa_{0}(\alpha)}\left(t-t_{j}\right)^{\alpha}\right) .
\end{aligned}
$$

\subsection{Fractional respiratory mechanics model through proportional derivative}

In the current segment, we introduce the respiratory mechanics model employing non-local fractional proportional derivative defined by the proportional derivative (PD) controller. The underlying fractional model can be given by the proportional derivative as follows

$$
\begin{gathered}
R\left[{ }_{P} \mathbb{D}^{\alpha, \rho} \mathbf{V}_{i}(t)\right]+\left(\frac{1}{C}\right) \mathbf{V}_{i}(t)+P_{m}=P_{d}, \quad 0 \leqslant t \leqslant t_{j}, \\
R\left[{ }_{P} \mathbb{D}^{\alpha, \rho} \mathbf{V}_{e}(t)\right]+\left(\frac{1}{C}\right) \mathbf{V}_{e}(t)+P_{m}=0, \quad t_{j} \leqslant t \leqslant t_{b}, \\
\mathbf{V}_{i}(0)=\mathbf{V}_{e}\left(t_{b}\right)=0, \\
\mathbf{V}_{i}\left(t_{j}\right)=\mathbf{V}_{e}\left(t_{j}\right)=\mathbf{V}_{T} .
\end{gathered}
$$

The equation (3.70) can be solved by the LT of fractional proportional derivative under the condition $\mathbf{V}_{\mathfrak{i}}(0)=0$ as follows

$$
\begin{gathered}
\mathcal{L}\left\{R\left[\mathrm{P}^{\alpha, \rho} \mathbf{V}_{\mathfrak{i}}(\mathrm{t})\right]\right\}+\mathcal{L}\left\{\left(\frac{1}{\mathrm{C}}\right) \mathbf{V}_{\mathfrak{i}}(\mathrm{t})\right\}=\mathcal{L}\left\{\mathrm{P}_{\mathrm{d}}-\mathrm{P}_{\mathrm{m}}\right\}, \\
(\rho s+1-\rho)^{\alpha} \mathcal{L}\left\{\mathbf{V}_{\mathfrak{i}}(\mathrm{t})\right\}-\rho(\rho s+1-\rho)^{\alpha-1} \mathbf{V}_{\mathfrak{i}}(0)+\left(\frac{1}{\mathrm{CR}}\right) \mathcal{L}\left\{\mathbf{V}_{\mathfrak{i}}(\mathrm{t})\right\}=\frac{\mathrm{P}_{\mathrm{d}}-\mathrm{P}_{\mathrm{m}}}{\mathrm{Rs}}, \\
\mathcal{L}\left\{\mathbf{V}_{\mathfrak{i}}(\mathrm{t})\right\}=\frac{\mathrm{P}_{\mathrm{d}}-\mathrm{P}_{\mathrm{m}}}{\mathrm{Rs}} \frac{\rho^{-\alpha}}{\left(s-\frac{\rho-1}{\rho}\right)^{\alpha}+\frac{1}{\mathrm{CR}}},
\end{gathered}
$$

and taking inverse LT of the last equation and utilizing convolution theorem, we get the solution below

$$
\mathbf{V}_{\mathfrak{i}}(t)=\left(\frac{P_{d}-P_{m}}{R}\right) \rho^{-\alpha} \int_{0}^{t} \mathbf{E}_{\alpha, \alpha}\left(-\frac{1}{C R}(t-\tau)^{\alpha}\right) e^{\frac{\rho-1}{\rho}(t-\tau)}(t-\tau)^{\alpha-1} d \tau,
$$

accordingly, under the condition $\mathbf{V}_{\mathfrak{i}}\left(t_{\mathfrak{j}}\right)=\mathbf{V}_{\mathrm{T}}$, the solution of the differential equation (3.70) can be obtained by the LT as

$$
\mathcal{L}\left\{\mathrm{R}\left[\mathrm{p} \mathbb{D}^{\alpha, \rho} \mathbf{V}_{\mathfrak{i}}(\mathrm{t})\right]\right\}+\mathcal{L}\left\{\left(\frac{1}{\mathrm{C}}\right) \mathbf{V}_{\mathfrak{i}}(\mathrm{t})\right\}=\mathcal{L}\left\{\mathrm{P}_{\mathrm{d}}-\mathrm{P}_{\mathrm{m}}\right\},
$$




$$
\begin{gathered}
(\rho s+1-\rho)^{\alpha} \mathcal{L}\left\{\mathbf{V}_{\mathfrak{i}}(t)\right\}-\rho(\rho s+1-\rho)^{\alpha-1} \mathbf{V}_{\mathrm{T}}+\left(\frac{1}{\mathrm{CR}}\right) \mathcal{L}\left\{\mathbf{V}_{\mathfrak{i}}(\mathrm{t})\right\}=\frac{\mathrm{P}_{\mathrm{d}}-\mathrm{P}_{\mathrm{m}}}{\mathrm{Rs}}, \\
\mathcal{L}\left\{\mathbf{V}_{\mathfrak{i}}(\mathrm{t})\right\}=\frac{\left(\mathrm{s}-\frac{\rho-1}{\rho}\right)^{\alpha-1} \mathbf{V}_{\mathrm{T}}}{\left(\mathrm{s}-\frac{\rho-1}{\rho}\right)^{\alpha}+\frac{1}{\mathrm{CR}}}+\frac{\mathrm{P}_{\mathrm{d}}-\mathrm{P}_{\mathrm{m}}}{\mathrm{Rs}} \frac{\rho^{-\alpha}}{\left(s-\frac{\rho-1}{\rho}\right)^{\alpha}+\frac{1}{\mathrm{CR}}},
\end{gathered}
$$

and with the inverse LT, we reach the desired result

$$
\begin{aligned}
\mathbf{V}_{\mathfrak{i}}(\mathbf{t}) & =\mathbf{V}_{\mathrm{T}} e^{\frac{\rho-1}{\rho}\left(t-t_{j}\right)}\left(t-t_{j}\right)^{\alpha-1} \mathbf{E}_{\alpha}\left(-\frac{1}{C R}\left(t-t_{j}\right)^{\alpha}\right) \\
& +\left(\frac{P_{d}-P_{m}}{R}\right) \rho^{-\alpha} \int_{t_{j}}^{t} E_{\alpha, \alpha}\left(-\frac{1}{C R}(t-\tau)^{\alpha}\right) e^{\frac{\rho-1}{\rho}(t-\tau)}(t-\tau)^{\alpha-1} d \tau .
\end{aligned}
$$

By following similar steps above, the equation (3.71) can be solved with the help of LT under the initial and end conditions $\mathbf{V}_{e}(t b)=0$ and $\mathbf{V}_{e}\left(t_{j}\right)=\mathbf{V}_{\mathrm{T}}$. Hence, we obtain the solutions of (3.71) with these conditions as follows, respectively

$$
\mathbf{V}_{e}(t)=-\frac{P_{m}}{R} \rho^{-\alpha} \int_{t_{b}}^{t} E_{\alpha, \alpha}\left(-\frac{1}{C R}(t-\tau)^{\alpha}\right) e^{\frac{\rho-1}{\rho}(t-\tau)}(t-\tau)^{\alpha-1} d \tau,
$$

and

$$
\begin{aligned}
\mathbf{V}_{e}(t) & =\mathbf{V}_{T} e^{\frac{\rho-1}{\rho}\left(t-t_{j}\right)}\left(t-t_{j}\right)^{\alpha-1} \mathbf{E}_{\alpha}\left(-\frac{1}{C R}\left(t-t_{j}\right)^{\alpha}\right) \\
& -\left(\frac{P_{m}}{R}\right) \rho^{-\alpha} \int_{t_{j}}^{t} E_{\alpha, \alpha}\left(-\frac{1}{C R}(t-\tau)^{\alpha}\right) e^{\frac{\rho-1}{\rho}(t-\tau)}(t-\tau)^{\alpha-1} d \tau .
\end{aligned}
$$

\subsection{Fractional respiratory mechanics model through generalized derivative}

The mechanical process performed by the ventilator can be model by generalized derivative including two parameters $\rho$ and $\alpha$. In this way, we obtain general fractional solutions with two parameters by making use of LT of generalized derivative introduced by Katugampola. The fractional respiratory mechanics model with generalized derivative can be presented by

$$
\begin{gathered}
R\left[\mathbb{D}^{\alpha, \rho} \mathbf{V}_{\mathfrak{i}}(t)\right]+\left(\frac{1}{C}\right) \mathbf{V}_{i}(t)+P_{m}=P_{d}, \quad 0 \leqslant t \leqslant t_{j}, \\
R\left[\mathbb{D}^{\alpha, \rho} \mathbf{V}_{e}(t)\right]+\left(\frac{1}{C}\right) \mathbf{V}_{e}(t)+P_{m}=0, \quad t_{j} \leqslant t \leqslant t_{b}, \\
\mathbf{V}_{\mathfrak{i}}(0)=\mathbf{V}_{e}\left(t_{b}\right)=0, \\
\mathbf{V}_{\mathfrak{i}}\left(t_{j}\right)=\mathbf{V}_{e}\left(t_{j}\right)=\mathbf{V}_{T} .
\end{gathered}
$$


Let us apply the LT to the both side of the equation (3.84) under the initial condition $\mathbf{V}_{\mathfrak{i}}(0)=0$, so we have

$$
\begin{gathered}
\mathcal{L}\left\{R\left[\mathbb{D}^{\alpha, \rho} \mathbf{V}_{\mathfrak{i}}(\mathrm{t})\right]\right\}+\mathcal{L}\left\{\left(\frac{1}{\mathrm{C}}\right) \mathbf{V}_{\mathfrak{i}}(\mathrm{t})\right\}=\mathcal{L}\left\{\mathrm{P}_{\mathrm{d}}-\mathrm{P}_{\mathrm{m}}\right\}, \\
\mathrm{s}^{\alpha} \mathcal{L}\left\{\mathbf{V}_{\mathfrak{i}}(\mathrm{t})\right\}-\mathrm{s}^{\alpha-1} \mathbf{V}_{\mathfrak{i}}(0)+\left(\frac{1}{\mathrm{CR}}\right) \mathcal{L}\left\{\mathbf{V}_{\mathfrak{i}}(\mathrm{t})\right\}=\frac{\mathrm{P}_{\mathrm{d}}-\mathrm{P}_{\mathrm{m}}}{\mathrm{Rs}}, \\
\mathcal{L}\left\{\mathbf{V}_{\mathfrak{i}}(\mathrm{t})\right\}=\frac{\mathrm{P}_{\mathrm{d}}-\mathrm{P}_{\mathrm{m}}}{\mathrm{R}} \frac{1}{\mathrm{~s}\left(\mathrm{~s}^{\alpha}+\frac{1}{\mathrm{CR}}\right)},
\end{gathered}
$$

taking inverse Lt of the last equation, one can easily get

$$
\mathbf{V}_{\mathfrak{i}}(\mathrm{t})=\mathrm{C}\left(\mathrm{P}_{\mathrm{d}}-\mathrm{P}_{\mathrm{m}}\right)\left[1-\mathbf{E}_{\alpha}\left(-\frac{1}{\mathrm{CR}}\left(\frac{\mathrm{t}^{\rho}}{\rho}\right)^{\alpha}\right)\right] \text {. }
$$

After solving the same equation with the end condition $\mathbf{V}_{\mathfrak{i}}\left(t_{j}\right)=\mathbf{V}_{\mathrm{T}}$, we obtain the following solution

$$
\begin{aligned}
\mathbf{V}_{i}(t) & =C\left(P_{d}-P_{m}\right)\left[1-E_{\alpha}\left(-\frac{1}{C R}\left(\frac{\left(t-t_{j}\right)^{\rho}}{\rho}\right)^{\alpha}\right)\right] \\
& +\mathbf{V}_{T}\left[E_{\alpha}\left(-\frac{1}{C R}\left(\frac{\left(t-t_{j}\right)^{\rho}}{\rho}\right)^{\alpha}\right)\right] .
\end{aligned}
$$

If we apply the LT of the generalized derivative to the equation (3.85) in the underlying model with the condition $\mathbf{V}_{\mathrm{e}}\left(\mathrm{t}_{\mathrm{b}}\right)=0$, we reach the results below

$$
\begin{gathered}
\mathcal{L}\left\{R\left[C \mathbb{D}^{\alpha} \mathbf{V}_{e}(t)\right]\right\}+\mathcal{L}\left\{\left(\frac{1}{\mathrm{C}}\right) \mathbf{V}_{e}(t)\right\}=\mathcal{L}\left\{-\mathrm{P}_{\mathrm{m}}\right\} \\
\mathrm{s}^{\alpha} \mathcal{L}\left\{\mathbf{V}_{\mathrm{e}}(\mathrm{t})\right\}-\mathrm{s}^{\alpha-1} \mathbf{V}_{\mathrm{e}}\left(\mathrm{t}_{\mathrm{b}}\right)+\left(\frac{1}{\mathrm{CR}}\right) \mathcal{L}\left\{\mathbf{V}_{\mathrm{e}}(\mathrm{t})\right\}=\frac{-\mathrm{P}_{\mathrm{m}}}{\mathrm{Rs}}, \\
\mathcal{L}\left\{\mathbf{V}_{\mathrm{e}}(\mathrm{t})\right\}=\frac{-\mathrm{P}_{\mathrm{m}}}{\mathrm{R}} \frac{1}{\mathrm{~s}\left(\mathrm{~s}^{\alpha}+\frac{1}{\mathrm{CR}}\right)},
\end{gathered}
$$

applying inverse LT to (3.95), one can obtain

$$
\mathbf{V}_{e}(t)=-\mathrm{CP}_{\mathrm{m}}\left[1-\mathbf{E}_{\alpha}\left(-\frac{1}{\mathrm{CR}}\left(\frac{\left(\mathrm{t}-\mathrm{t}_{\mathrm{b}}\right)^{\rho}}{\rho}\right)^{\alpha}\right)\right] .
$$

Similarly, for the equation (3.85) under the end condition $\mathbf{V}_{\boldsymbol{e}}\left(\mathbf{t}_{\mathbf{j}}\right)=\mathbf{V}_{\mathrm{T}}$, we conveniently attain the following solution

$$
\begin{aligned}
\mathbf{V}_{e}(\mathrm{t}) & =-\mathrm{CP}_{\mathrm{m}}\left[1-\mathbf{E}_{\alpha}\left(-\frac{1}{\mathrm{CR}}\left(\frac{\left(\mathrm{t}-\mathrm{t}_{j}\right)^{\rho}}{\rho}\right)^{\alpha}\right)\right] \\
& +\mathbf{V}_{\mathrm{T}}\left[\mathbf{E}_{\alpha}\left(-\frac{1}{\mathrm{CR}}\left(\frac{\left(\mathrm{t}-\mathrm{t}_{j}\right)^{\rho}}{\rho}\right)^{\alpha}\right)\right] .
\end{aligned}
$$




\subsection{Fractional respiratory mechanics model through M-derivative}

The instantaneous volume of the lung can be observed by fractional respiratory mechanics model including M-derivative with three parameters $\alpha, \rho$ and $\gamma$. This fractional operator enable us to get more general solutions than other operators given above. Thus we inspire to solve the underlying model in terms of the non-local fractional truncated M-derivative. Fractional respiratory mechanics model can be given by

$$
\begin{gathered}
R\left[{ }_{M} \mathbb{D}^{\alpha, \rho, \gamma} \mathbf{V}_{i}(t)\right]+\left(\frac{1}{C}\right) \mathbf{V}_{i}(t)+P_{m}=P_{d}, \quad 0 \leqslant t \leqslant t_{j}, \\
R\left[M \mathbb{D}^{\alpha, \rho, \gamma} \mathbf{V}_{e}(t)\right]+\left(\frac{1}{C}\right) \mathbf{V}_{e}(t)+P_{m}=0, \quad t_{j} \leqslant t \leqslant t_{b}, \\
\mathbf{V}_{\mathfrak{i}}(0)=\mathbf{V}_{e}\left(t_{b}\right)=0, \\
\mathbf{V}_{\mathfrak{i}}\left(t_{j}\right)=\mathbf{V}_{e}\left(t_{j}\right)=\mathbf{V}_{T} .
\end{gathered}
$$

If we solve the differential equation (3.98) with the initial condition $\mathbf{V}_{\mathfrak{i}}(0)=0$ by virtue of the LT of fractional M-derivative, then we can get

$$
\begin{gathered}
\mathcal{L}\left\{R\left[M \mathbb{D}^{\alpha, \rho, \gamma} \mathbf{V}_{\mathfrak{i}}(\mathrm{t})\right]\right\}+\mathcal{L}\left\{\left(\frac{1}{\mathrm{C}}\right) \mathbf{V}_{\mathfrak{i}}(\mathrm{t})\right\}=\mathcal{L}\left\{\mathrm{P}_{\mathrm{d}}-\mathrm{P}_{\mathrm{m}}\right\}, \\
\mathrm{s}^{\alpha} \mathcal{L}\left\{\mathbf{V}_{\mathfrak{i}}(\mathrm{t})\right\}-\mathrm{s}^{\alpha-1} \mathbf{V}_{\mathfrak{i}}(0)+\left(\frac{1}{\mathrm{CR}}\right) \mathcal{L}\left\{\mathbf{V}_{\mathfrak{i}}(\mathrm{t})\right\}=\frac{\mathrm{P}_{\mathrm{d}}-\mathrm{P}_{\mathrm{m}}}{\mathrm{Rs}}, \\
\mathcal{L}\left\{\mathbf{V}_{\mathfrak{i}}(\mathrm{t})\right\}=\frac{\mathrm{P}_{\mathrm{d}}-\mathrm{P}_{\mathrm{m}}}{\mathrm{R}} \frac{1}{\mathrm{~s}\left(\mathrm{~s}^{\alpha}+\frac{1}{\mathrm{CR}}\right)},
\end{gathered}
$$

and by taking inverse LT, one can have

$$
\mathbf{V}_{\mathfrak{i}}(\mathrm{t})=\mathrm{C}\left(\mathrm{P}_{\mathrm{d}}-\mathrm{P}_{\mathrm{m}}\right)\left[1-\mathbf{E}_{\alpha}\left(-\frac{1}{\mathrm{CR}}\left(\Gamma(\gamma+1) \frac{\mathrm{t}^{\rho}}{\rho}\right)^{\alpha}\right)\right]
$$

Also, with the condition $\mathbf{V}_{\mathfrak{i}}\left(\mathbf{t}_{\mathfrak{j}}\right)=\mathbf{V}_{\mathrm{T}}$ for (3.98), we obtain the solution as

$$
\begin{aligned}
\mathbf{V}_{\mathrm{i}}(\mathrm{t}) & =\mathrm{C}\left(\mathrm{P}_{\mathrm{d}}-\mathrm{P}_{\mathrm{m}}\right)\left[1-\mathbf{E}_{\alpha}\left(-\frac{1}{\mathrm{CR}}\left(\Gamma(\gamma+1) \frac{\left(\mathrm{t}-\mathrm{t}_{\mathrm{j}}\right)^{\rho}}{\rho}\right)^{\alpha}\right)\right] \\
& +\mathbf{V}_{\mathrm{T}}\left[\mathbf{E}_{\alpha}\left(-\frac{1}{\mathrm{CR}}\left(\Gamma(\gamma+1) \frac{\left(\mathrm{t}-\mathrm{t}_{\mathrm{j}}\right)^{\rho}}{\rho}\right)^{\alpha}\right)\right] .
\end{aligned}
$$

On the other part, let us compute the solution of the equation (3.99) under the condition $\mathbf{V}_{e}\left(t_{b}\right)=0$ with the help of LT as follows

$$
\begin{gathered}
\mathcal{L}\left\{R\left[C \mathbb{D}^{\alpha} \mathbf{V}_{e}(t)\right]\right\}+\mathcal{L}\left\{\left(\frac{1}{C}\right) \mathbf{V}_{e}(t)\right\}=\mathcal{L}\left\{-P_{m}\right\}, \\
s^{\alpha} \mathcal{L}\left\{\mathbf{V}_{e}(t)\right\}-s^{\alpha-1} \mathbf{V}_{e}\left(t_{b}\right)+\left(\frac{1}{C R}\right) \mathcal{L}\left\{\mathbf{V}_{e}(t)\right\}=\frac{-P_{m}}{R s},
\end{gathered}
$$




$$
\mathcal{L}\left\{\mathbf{V}_{\mathrm{e}}(\mathrm{t})\right\}=\frac{-\mathrm{P}_{\mathrm{m}}}{\mathrm{R}} \frac{1}{\mathrm{~s}\left(\mathrm{~s}^{\alpha}+\frac{1}{\mathrm{CR}}\right)},
$$

applying inverse LT, we get

$$
\mathbf{V}_{e}(t)=-C P_{m}\left[1-\mathbf{E}_{\alpha}\left(-\frac{1}{C R}\left(\Gamma(\gamma+1) \frac{\left(t-t_{b}\right)^{\rho}}{\rho}\right)^{\alpha}\right)\right],
$$

and similarly with the condition $\mathbf{V}_{e}\left(t_{j}\right)=\mathcal{V}_{T}$, one can readily attain the solution below

$$
\begin{aligned}
\mathbf{V}_{\mathrm{e}}(\mathrm{t}) & =-\mathrm{CP}_{\mathrm{m}}\left[1-\mathbf{E}_{\alpha}\left(-\frac{1}{\mathrm{CR}}\left(\Gamma(\gamma+1) \frac{\left(\mathrm{t}-\mathrm{t}_{\mathrm{j}}\right)^{\rho}}{\rho}\right)^{\alpha}\right)\right] \\
& +\mathbf{V}_{\mathrm{T}}\left[\mathbf{E}_{\alpha}\left(-\frac{1}{\mathrm{CR}}\left(\Gamma(\gamma+1) \frac{\left(\mathrm{t}-\mathrm{t}_{\mathrm{j}}\right)^{\rho}}{\rho}\right)^{\alpha}\right)\right] .
\end{aligned}
$$

\section{Discussions and Concluding remarks}

In this study, the mechanical process model performed by the ventilator has been solved with some non-local fractional derivatives and analyzed in detail. With this rigorous analysis, non-local derivatives have been handled separately and similar and different aspects have been shown with each other. With the help of the graphs, we have pointed out the behavior of each derivative by changing the parameter values and observed by the movement of the curves on the graphs. Moreover, since we are working on one, two and three-parameter derivatives from non-local fractional operators in the literature, the effect of increasing parameters on solution curves is clearly seen. The closeness or distance of the solution curves to the classical derivative for different parameter values of $\alpha, \gamma$, and $\rho$ can be clearly observed on the graphs. Here the classical derivative corresponds to $\alpha=1, \gamma=1$ and $\rho=1$. We have drawn all graphics for $\mathrm{R}=10 \mathrm{~cm}\left(\mathrm{H}_{2} \mathrm{O}\right) / \mathrm{L} / \mathrm{sec}, \mathrm{C}=0.02 \mathrm{~L} / \mathrm{cm}\left(\mathrm{H}_{2} \mathrm{O}\right) \mathrm{P}_{\mathrm{d}}=20 \mathrm{~cm}\left(\mathrm{H}_{2} \mathrm{O}\right), \mathrm{t}_{\mathrm{j}}=1 \mathrm{sec}$ and $\mathrm{t}_{\mathrm{b}}=3 \mathrm{sec}$ values and we have compared fractional derivatives separately with the classical derivative. In Figures 1, 2 and 3, the Caputo derivative has been compared for the values of $\alpha=1,0.65,0.45,0.25, \alpha=1,0.99,0.98,0.97$ and $\alpha=1,0.9,0.8,0.7$, respectively. Also, in Figure 4 we have carried out the comparison for $\rho=1,0.9,0.8,0.7$ when $\alpha=0.9$ and in Figure 5, 6 similar comparison performed for $\alpha=1,0.9,0.8,0.7$ when $\rho=0.6$ and $\rho=0.9$, respectively. Figure 7 shows the solutions curves for $\alpha=1,0.99,0.98,0.978$ in the frame of generalized fractional derivative. We have observed the behavior of the solutions curves when $\gamma=1,1.5,2.5,3.5$ in Figure 8 and so we can see clearly the effect of the $\gamma$ parameter on the solutions. In Figures 9 and 10, the solutions curves have been showed for non-local fractional M-derivative when $\alpha=1,0.9,0.8,0.7$ and $\gamma=1,1.2,1.25,1.3$. In addition, we make a comparison for fractional proportional derivative for different values of $\alpha$ in Figures 11 and 12. Lastly, in Figures 13 and 14, the above-stated four fractional operators and classical derivative have been compared with each other. Employing different types of fractional derivatives, different solution steps, and solution curves have been obtained for the fractional respiratory mechanics model under investigation in this study. Therefore, the results enable us to analyze the 
same class of non-local fractional operators with a singular kernel. In a future study, it can be expressed which fractional derivative is more suitable and useful for the underlying model if real-life data are utilized. The aim of this study is to provide alternative fractional solutions for future studies and also to provide the opportunity to observe the patient in more detail through the fractional derivatives with memory-effect if the mechanical process model performed by the ventilator is used in patient control. In this way, some advantages may arise to prevent possible dangers caused by the lack of monitoring patients. On the other hand, it is worth mentioning that the proposed model has been examined and analyzed with non-local fractional operators for the first time in this study.
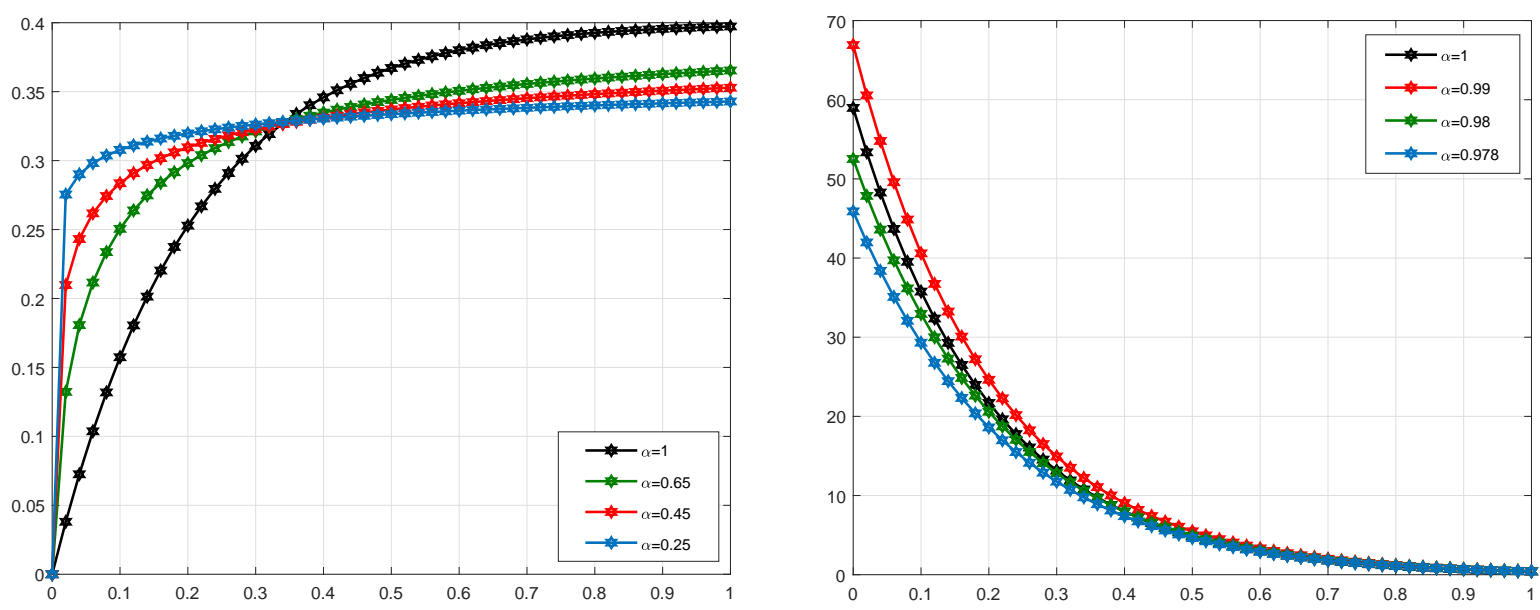

Figure 1: Comparison with Caputo derivative for $V_{i}(t)$
Figure 2: Comparison with Caputo derivative for $V_{e}(t)$ 

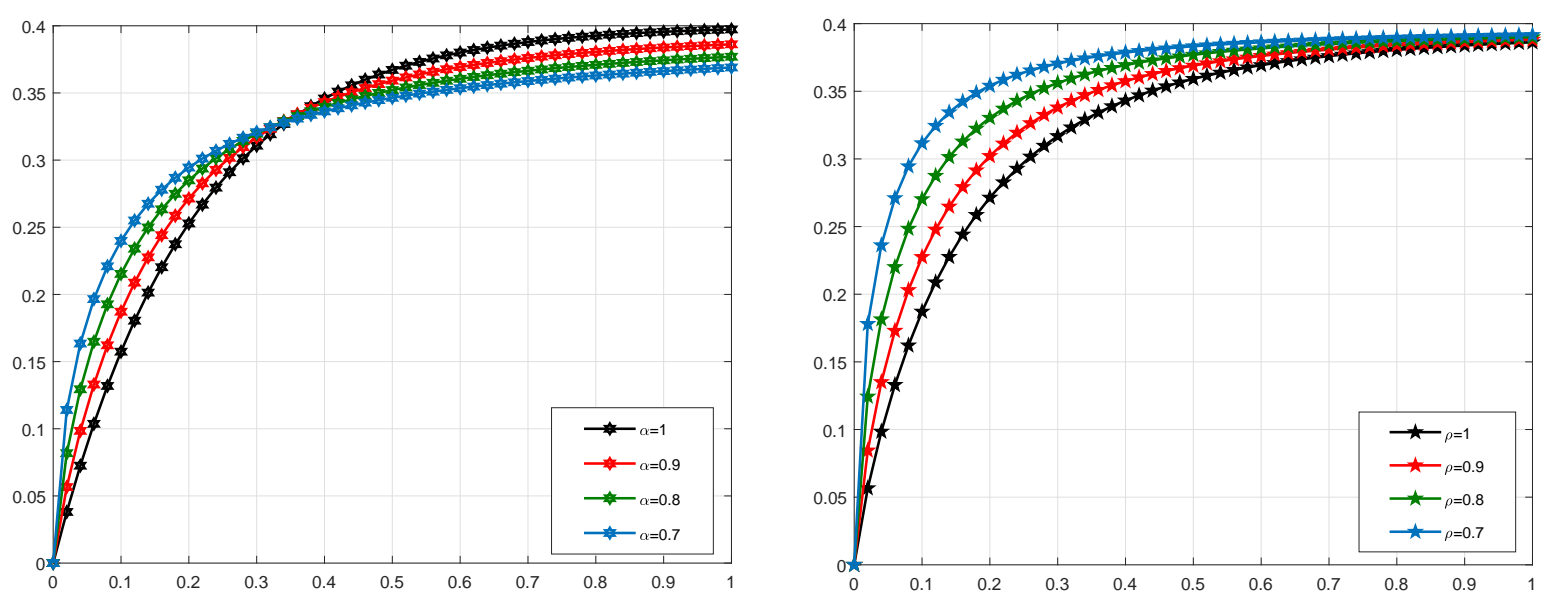

Figure 3: Comparison with Caputo derivative for $\mathbf{V}_{i}(t)$
Figure 4: Comparison with generalized derivative for $\mathbf{V}_{\mathfrak{i}}(\mathbf{t})$

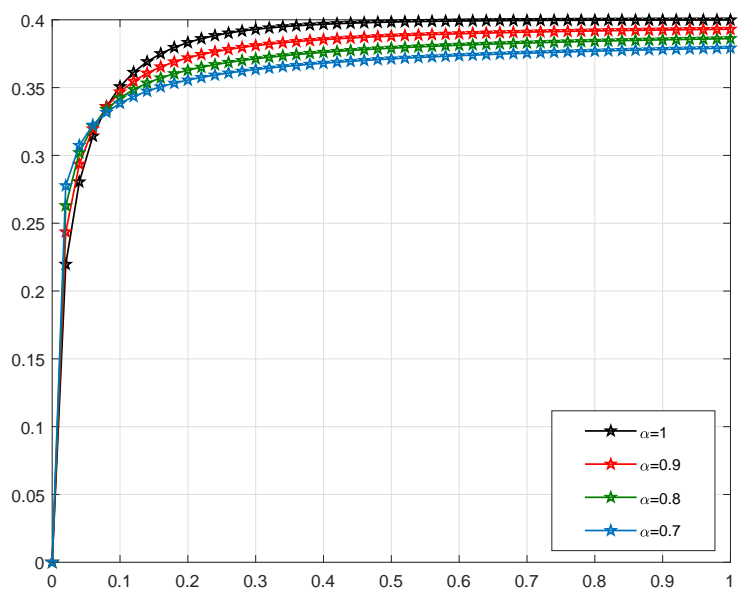

Figure 5: Comparison with generalized derivative for $\mathbf{V}_{\mathfrak{i}}(\mathrm{t})$

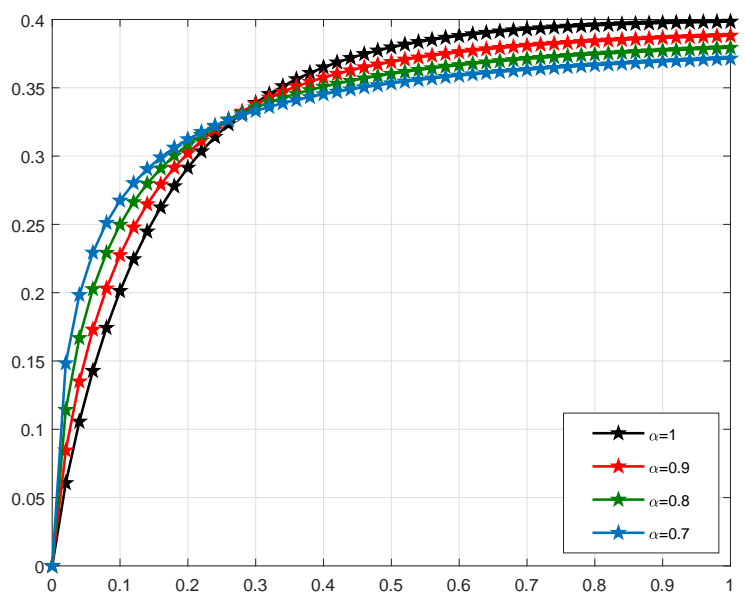

Figure 6: Comparison with generalized derivative for $\mathbf{V}_{\boldsymbol{i}}(\mathbf{t})$ 

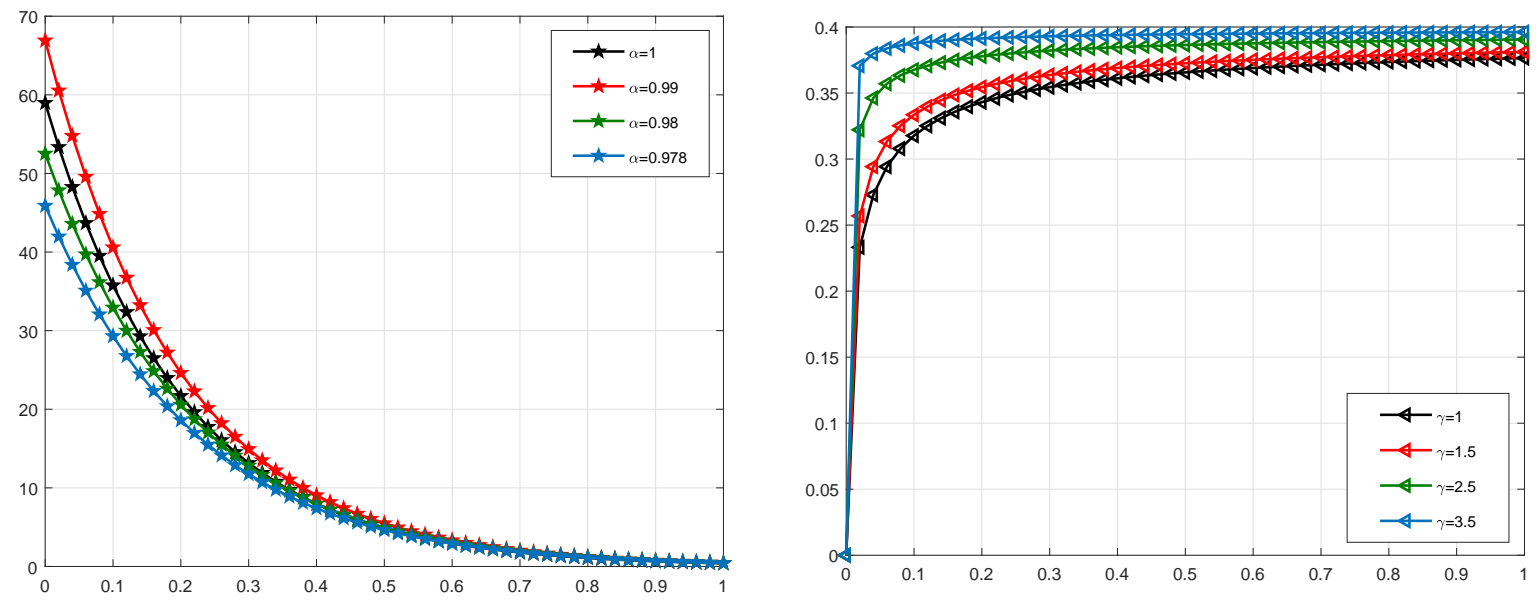

Figure 7: Comparison with generalized derivative for $\mathbf{V}_{e}(\mathbf{t})$
Figure 8: Comparison with non-local $M-$ derivative for $\mathbf{V}_{i}(t)$

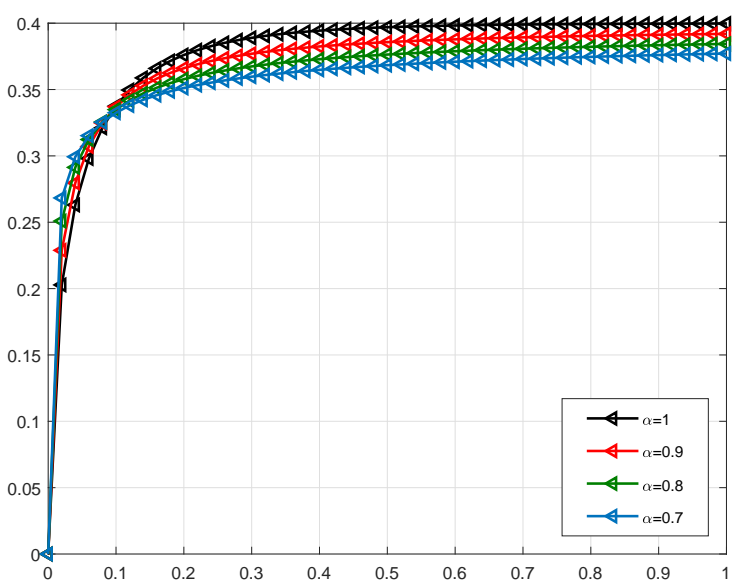

Figure 9: Comparison with non-local $M-$ derivative for $\mathbf{V}_{\boldsymbol{i}}(\mathbf{t})$

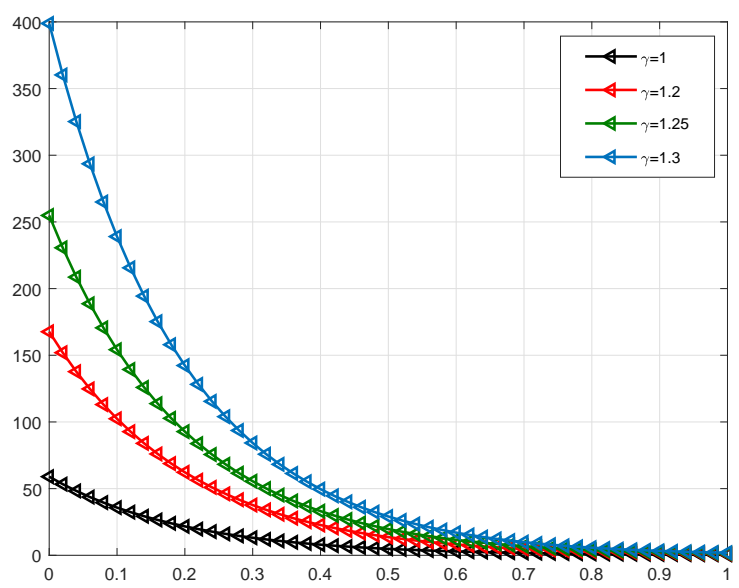

Figure 10: Comparison with non-local $M$ derivative for $\mathbf{V}_{e}(t)$ 

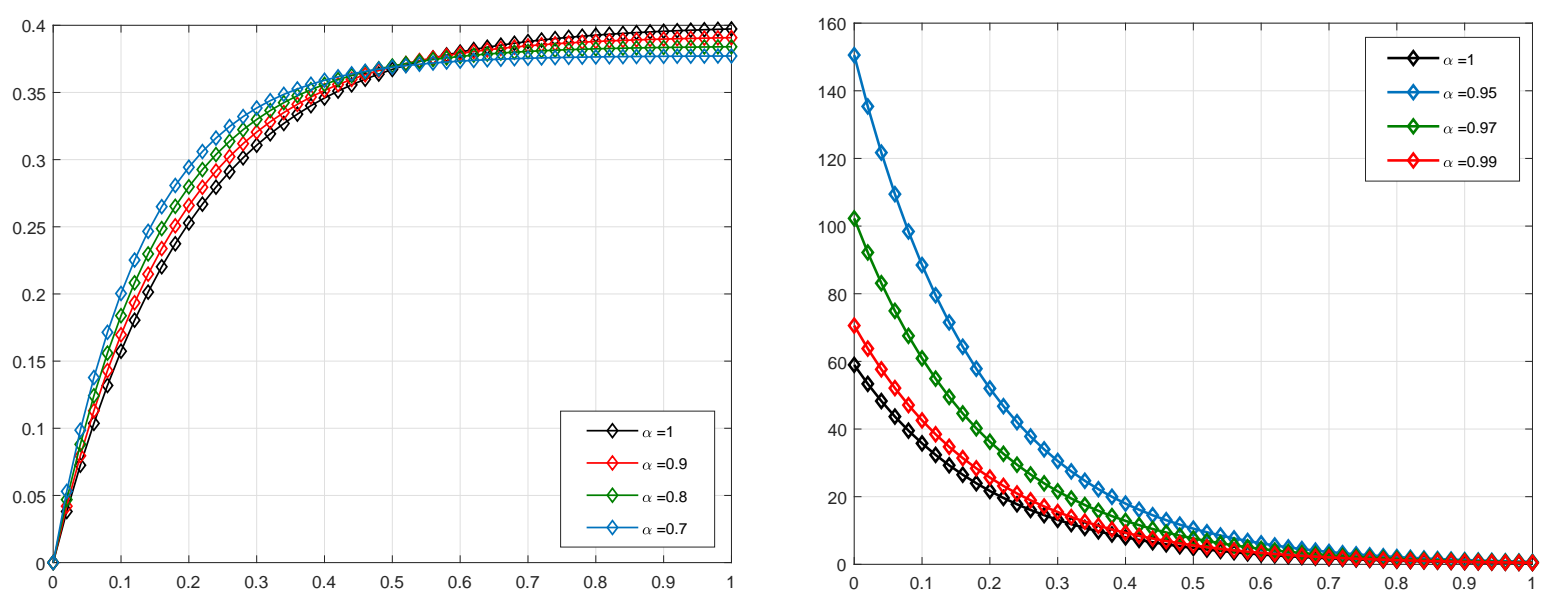

Figure 11: Comparison with non-local proportional derivative for $\mathbf{V}_{\mathfrak{i}}(\mathrm{t})$
Figure 12: Comparison with non-local proportional derivative for $\mathbf{V}_{e}(\mathrm{t})$

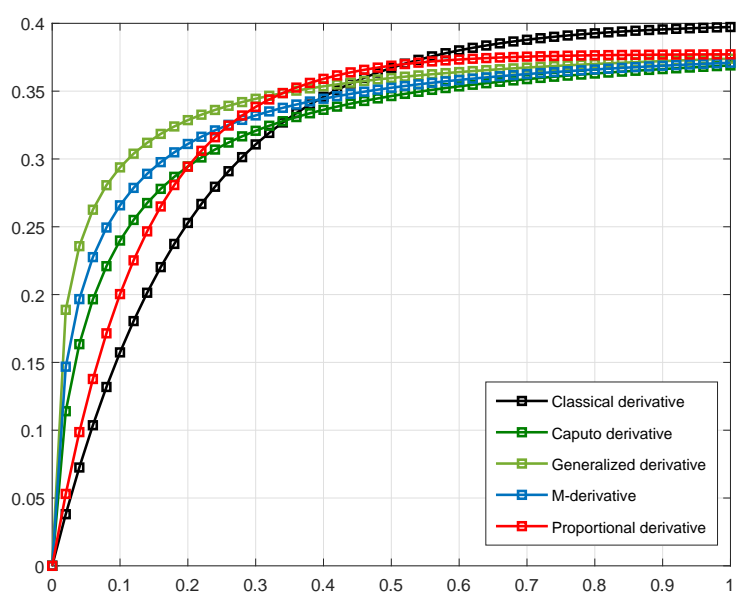

Figure 13: Comparison for $\mathbf{V}_{\mathfrak{i}}(\mathbf{t})$

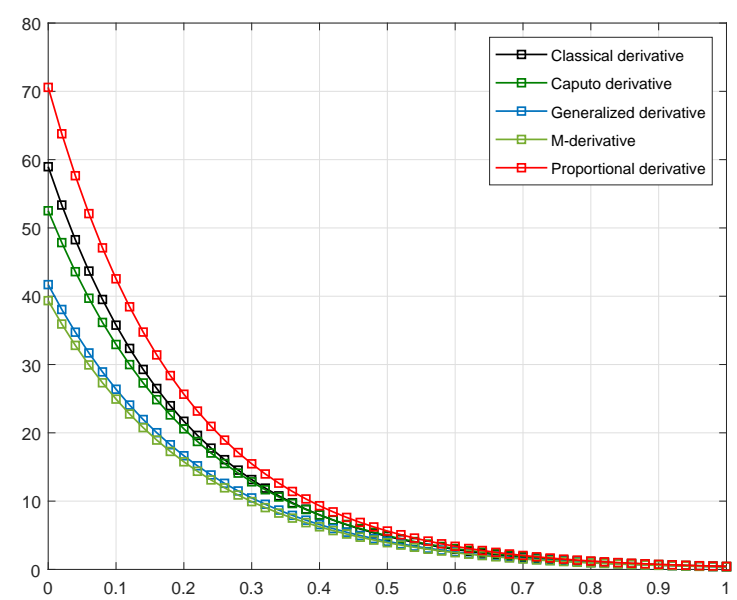

Figure 14: Comparison for $\mathbf{V}_{e}(t)$ 
Data Availability: All data used for the findings in this research are available publicly in the manuscript.

\section{References}

[1] Kilbas AA, Srivastava HM, and Trujillo JJ (2006). "Theory and Applications of Fractional Differential Equations". North-Holland Math Stud, Elsevier, Amsterdam, 204.

[2] Fernandez, A., Ozarslan, M. A., Baleanu, D. (2019). On fractional calculus with general analytic kernels. Applied Mathematics and Computation, 354, 248-265. https://doi.org/10.1016/j.amc.2019.02.045

[3] Jarad, F., Abdeljawad, T., Alzabut, J. (2017). Generalized fractional derivatives generated by a class of local proportional derivatives. The European Physical Journal Special Topics, 226(16-18), 3457-3471. https:/ / doi.org/10.1140/epjst/e2018-00021-7

[4] Baleanu, D., Fernandez, A., Akgül, A. (2020). On a Fractional Operator Combining Proportional and Classical Differintegrals. Mathematics, 8(3), 360. https://doi.org/10.3390/math8030360

[5] Anderson, D. R., Ulness, D. J. (2015). Newly defined conformable derivatives. Adv. Dyn. Syst. Appl, 10(2), 109-137. http:/ / campus.mst.edu/adsa

[6] Prabhakar, T. R. (1971). A singular integral equation with a generalized Mittag Leffler function in the kernel.

[7] Kilbas, A. A., Saigo, M., Saxena, R. K. (2004). Generalized Mittag-Leffler function and generalized fractional calculus operators. Integral Transforms and Special Functions, 15(1), 31-49. https:/ /doi.org/10.1080/10652460310001600717

[8] Acay, B., Bas, E., Abdeljawad, T. (2020). Non-local fractional calculus from different viewpoint generated by truncated M-derivative. Journal of Computational and Applied Mathematics, 366, 112410. https:/ / doi.org/10.1016/j.cam.2019.112410

[9] Katugampola, U. N. (2011). New approach to a generalized fractional integral. Applied Mathematics and Computation, 218(3), 860-865. https:/ / doi.org/10.1016/j.amc.2011.03.062

[10] Atangana, A., Baleanu, D. (2016). New fractional derivatives with nonlocal and non-singular kernel: theory and application to heat transfer model. arXiv preprint arXiv:1602.03408.

[11] Baleanu, D., Fernandez, A. (2019). On fractional operators and their classifications. Mathematics, 7(9), 830. https://doi.org/10.3390/math7090830

[12] Qureshi, S., Yusuf, A., Shaikh, A. A., Inc, M., Baleanu, D. (2019). Fractional modeling of blood ethanol concentration system with real data application. Chaos: An Interdisciplinary Journal of Nonlinear Science, 29(1), 013143. https:/ / doi.org/10.1063/1.5082907

[13] Qureshi, S., Yusuf, A. (2019). Modeling chickenpox disease with fractional derivatives: From caputo to atangana-baleanu. Chaos, Solitons \& Fractals, 122, 111-118. https:/ / doi.org/10.1016/j.chaos.2019.03.020

[14] Qureshi, S., Yusuf, A. (2019). Mathematical modeling for the impacts of deforestation on wildife species using Caputo differential operator. Chaos, Solitons \& Fractals, 126, 32-40. https:/ / doi.org/10.1016/j.chaos.2019.05.037

[15] Acay, B., Bas, E., Abdeljawad, T. (2020). Fractional economic models based on market equilibrium in the frame of different type kernels. Chaos, Solitons Fractals, 130, 109438. https://doi.org/10.1016/j.chaos.2019.109438

[16] Bas, E., Acay, B., Ozarslan, R. (2019). The price adjustment equation with different types of conformable derivatives in market equilibrium. https://doi.org/10.3934/math.2019.3.805

[17] Acay, B., Ozarslan, R., Bas, E. (2020). Fractional physical models based on falling body problem. AIMS Mathematics, 5(3), 2608. https://doi.org/10.3934/math.2020170

[18] Yavuz, M. (2017). Novel solution methods for initial boundary value problems of fractional order with conformable differentiation. An International Journal of Optimization and Control: Theories Applications (IJOCTA), 8(1), 1-7. https://doi.org/10.11121/ijocta.01.2018.00540

[19] Yusuf, A., Inc, M., Aliyu, A. I. (2018). On dark optical solitons of the space time nonlinear Schrödinger equation with fractional complex transform for Kerr and power law nonlinearities. Journal of Coupled Systems and Multiscale Dynamics, 6(2), 114-120. https://doi.org/10.1166/jcsmd.2018.1154

[20] Ozarslan, R. (2020). Microbial survival and growth modeling in frame of nonsingular fractional derivatives. Mathematical Methods in the Applied Sciences. https://doi.org/10.1002/mma.6357

[21] Yavuz, M., Yokus, A. Analytical and numerical approaches to nerve impulse model of fractional-order. Numerical Methods for Partial Differential Equations. https:/ /doi.org/10.1002/num.22476 
[22] Khan, A., Gómez-Aguilar, J. F., Khan, T. S., \& Khan, H. (2019). Stability analysis and numerical solutions of fractional order HIV/AIDS model. Chaos, Solitons \& Fractals, 122, 119-128. https:/ / doi.org/10.1016/j.chaos.2019.03.022

[23] Khan, A., Li, Y., Shah, K., \& Khan, T. S. (2017). On coupled-Laplacian fractional differential equations with nonlinear boundary conditions. Complexity, 2017. https:/ / doi.org/10.1155/2017/8197610

[24] Khan, H., Tunc, C., \& Khan, A. (2020). Green function's properties and existence theorems for nonlinear singular-delay-fractional differential equations. Discrete \& Continuous Dynamical Systems-S, 13(9), 2475. https:/ / doi.org/10.3934/dcdss.2020139

[25] Khan, H., Gomez-Aguilar, J. F., Abdeljawad, T., \& Khan, A. (2020). Existence results and stability criteria for ABC-fuzzy-Volterra integro-differential equation. Fractals, 2040048. https://doi.org/10.1142/S0218348X20400484

[26] Khan, H., Khan, Z. A., Tajadodi, H., \& Khan, A. (2020). Existence and data-dependence theorems for fractional impulsive integro-differential system. Advances in Difference Equations, 2020(1), 1-11. https://doi.org/10.1186/s13662-020-02823-6

[27] Liang, S., Wu, R., Chen, L. (2015). Laplace transform of fractional order differential equations. Electron. J. Differ. Equ, 139, 1-15.

[28] Jarad, F., Abdeljawad, T. (2018). A modifi ed Laplace transform for certain generalized fractional operators. Results in Nonlinear Analysis, 1(2), 88-98.

[29] Nagle, R. K., Saff, E. B., Snider, A. D. (2014). Fundamentals of differential equations. Pearson Education.

[30] Kürt, C., Özarslan, M. A., Fernandez, A. On a certain bivariate Mittag-Leffler function analysed from a fractional-calculus point of view. Mathematical Methods in the Applied Sciences.https:/ / doi.org/10.1002/mma.6324 